\title{
A MATHEMATICAL FRAMEWORK FOR DEVELOPING FREEZING PROTOCOLS IN THE CRYOPRESERVATION OF CELLS
}

\author{
MOHIT P. DALWADI*, SARAH L. WATERS*, HELEN M. BYRNE*, AND IAN J. HEWITT*
}

Abstract. When cooling cells to preserve them during cryopreservation, cooling too quickly results in the formation of lethal intracellular ice, while cooling too slowly amplifies the toxic effects of the cryoprotective agents (CPA) added to slow down ice formation. We derive a mathematical model for cell cryopreservation to understand and quantify these observations. We assume that the system has a spherical geometry of three different regions: ice, extracellular liquid medium, and cell. The two interfacial boundaries separating the three regions can move and must be determined as part of the solution. The presence of CPA lowers the freezing point of the system, and the cell membrane moves due to the osmotic pressure difference across the membrane. We use a combination of numerical and asymptotic methods to determine how the temperature, the CPA concentration, and concentration of an ion species internal and external to the cell evolve during cooling for a range of cooling rates across different timescales. We introduce two metrics to characterize the cell damage caused by freezing, accounting for supercooling and CPA toxicity. Given cell properties and the operating protocol of the cryopreservation process, we show how the damage metrics can be used to predict an optimal cooling rate. Our asymptotic analysis provides a computationally efficient framework from which to determine this optimal rate.

Key words. cryopreservation, supercooling, moving boundary problem, Stefan problem, asymptotic analysis

AMS subject classifications. 80A20, 80A22, 80M20, 80M35

1. Introduction. Cryopreservation is the process of preserving biological entities by cooling to temperatures low enough to halt biochemical processes such as metabolism [34, 37, 41]. This technology has a variety of uses, including fertility [31], tissue transplantation [25], food security [5], and the protection of endangered species [21]. While the exact details of cryopreservation protocols vary greatly between different cell types, unifying elements are the immersion of cells within a physiological liquid extracellular medium, and the subsequent cooling of this mixture [35]. It is imperative to be able to control and minimize intracellular ice formation during cryopreservation, which can be lethal to cells due to crushing or piercing of the cell from ice crystals [34, 39].

To combat ice formation, cryoprotective agents (CPAs), such as dimethyl sulphoxide (DMSO) or glycerol, are often added to the cryopreservation medium before cooling [18]. CPAs lower the freezing point of the cytosol and the medium, by interfering with the process by which crystalline ice structures form. As such, these CPAs must be able to permeate through the cell membrane. At the same time, the addition of CPA is not a panacea since CPAs can be toxic to cells at warmer temperatures, before the cooling process is complete [17]. Since intracellular ice formation is observed for faster cooling rates [33], and CPA toxicity is observed for slower cooling rates [17], a careful balance between CPA addition and cooling rate is required if the frozen cells are to remain viable. Typically the balance between these experimentally controllable parameters will be application specific and protocols are determined empirically [35, 36]. Mathematical and computational methods to simulate the cryopreservation process offer a cost-effective way to understand, refine, and optimize these protocols [1, 49].

The Kedem-Katchalsky (KK) equations are widely used in cryopreservation modelling to track cell volume and CPA concentration during cryopreservation [26, 29]. The KK equations consist of a system of coupled ordinary differential equations for the cell volume and the CPA concentration within the cell. The cell volume can vary during cryopreservation because osmotic pressures are generated across the membrane. This is caused by the concentrating of chemical species already present outside the cell as the available liquid volume decreases due to the growth of extracellular ice. However, as noted previously [1], the spatial variation of important physical quantities, such as the location of the freezing front and the CPA concentration through the system, are not typically taken into account in the KK equations. Although there are some exceptions (see, for example, $[8,16,24]$ ), most currently available spatial models only consider some aspect of spatial dependence, and rely on numerical simulations to solve them. The aim of this paper is to provide a model that accounts for the spatial dependence of temperature and chemical concentration and to systematically deduce the conditions under which simpler ODE models are applicable. This provides a mathematical framework in which optimal cooling rates for different cells can be deduced.

*Mathematical Institute, University of Oxford, Radcliffe Observatory Quarter, Oxford, OX2 6GG, UK (dalwadi@maths.ox.ac.uk) 

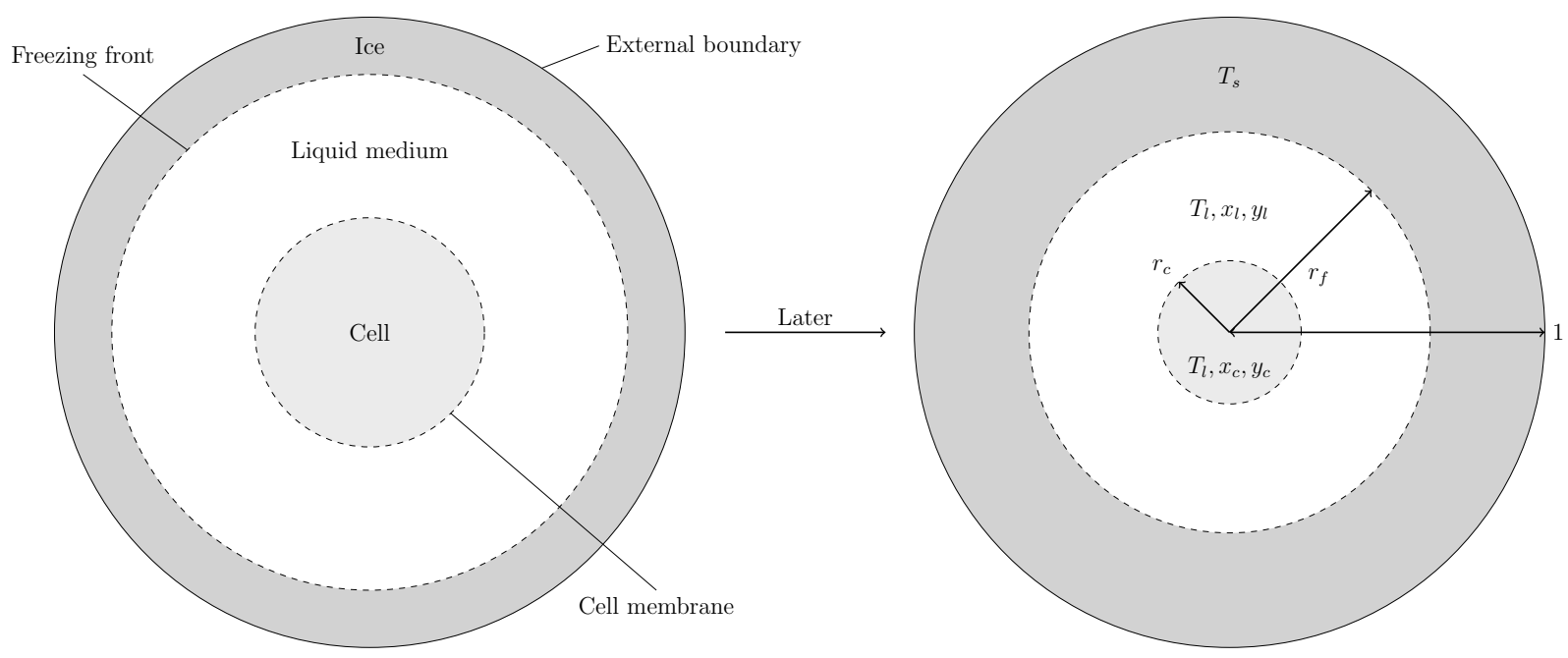

FIG. 1. A two-dimensional schematic of the three-dimensional spherically symmetric model geometry we consider in this paper. The dimensionless variables in each phase are defined in Table 1 . The cell membrane and freezing front, $r_{c}$ and $r_{f}$, respectively, are moving boundaries and are shown in dashed lines.

Three recent papers have investigated similar models to the one we present here [2, 3, 4]. In [2], a general model for the heat and mass transport around a cell surrounded by a liquid medium containing multiple chemical species is developed, taking into account spatial variation. As the system is cooled, a freezing front advances towards the cell. In [3], a version of this system is solved numerically for the case with a dilute and ideal ternary solution in a spherical domain, including the effects of solute capture within the ice, a pressure-dependent freezing point depression, and a Gibbs-Thomson freezing point promotion. In [4], numerical methods for solving the model in [3] are presented, and the effect of partial solute rejection at the ice-water interface is investigated. The model we consider in this paper is similar to those considered in $[3,4]$, however our approaches to analysing the models are different. We focus on obtaining asymptotic solutions to our model, which allow us to derive reduced equations that effectively govern the system, and yield significant physical insight into the heat and mass transport processes.

We consider the cryopreservation of a single cell in a liquid extracellular medium. The geometry, shown in Figure 1, consists of three different regions: ice, extracellular liquid, and cell liquid. The temperature of the external boundary is lowered at an operationally determined rate, and we solve for the temperature and concentrations of chemical species within the liquid medium and the cell. We track two representative species: one which can permeate the cell membrane, and one which cannot. As CPA is chosen for its ability to permeate cells, we refer to the permeable solute as CPA. As ions typically have a very low permeability across the cell membrane, we refer to the impermeable solute as the ion species. The concentration difference of these solutes across the membrane drives an osmotic liquid motion, and an associated change of cell volume. Additionally, as the exterior temperature decreases and ice forms, a freezing front will develop and propagate into the liquid phase. We therefore have two moving boundaries to track: the cell membrane and the freezing front.

We solve the resulting model using a combination of numerical and asymptotic methods. The latter allows us to systematically reduce the complexity of the model through the method of matched asymptotic expansions [20, 27], by exploiting an inherent separation of the natural timescales in the problem. These timescales are associated with heat conduction (seconds), chemical diffusion (minutes), and cell membrane movement (hours). Our approach allows us to identify the operating regimes where specific spatial effects of the temperature and chemical concentration are important, resulting in a comprehensive understanding of the possible cooling behaviours. Our asymptotic results also unveil the flow of information through the system and inform how we implement the relevant boundary conditions in our numerical scheme of the full problem.

Our asymptotically reduced model allows for a significant reduction in the computational complexity of 


\begin{tabular}{lll} 
Dimensional variable & Description & Dimensionless variable \\
\hline$\tilde{r}$ & Radial coordinate & $\tilde{r}=\tilde{r}_{b} r$ \\
$\tilde{t}$ & Time & $\tilde{t}=\left(\tilde{\rho}_{s} \tilde{c}_{s} \tilde{r}_{b}^{2} / \tilde{k}_{s}\right) t$ \\
$\tilde{x}_{c}$ & Intracellular CPA concentration & $\tilde{x}_{c}=\tilde{X}_{0} x_{c}$ \\
$\tilde{y}_{c}$ & Intracellular ion concentration & $\tilde{y}_{c}=\tilde{X}_{0} y_{c}$ \\
$\tilde{x}_{l}$ & Extracellular CPA concentration & $\tilde{x}_{l}=\tilde{X}_{0} x_{l}$ \\
$\tilde{y}_{l}$ & Extracellular ion concentration & $\tilde{y}_{l}=\tilde{X}_{0} y_{l}$ \\
$\tilde{T}_{l}$ & Water temperature & $\tilde{T}_{l}=\tilde{T}_{f 0}+\left(\tilde{T}_{f 0}-\tilde{T}_{\text {end }}\right) T_{l}$ \\
$\tilde{T}_{s}$ & Ice temperature & $\tilde{T}_{s}=\tilde{T}_{f 0}+\left(\tilde{T}_{f 0}-\tilde{T}_{\text {end }}\right) T_{s}$ \\
$\tilde{r}_{c}$ & Cell membrane & $\tilde{r}_{c}=\tilde{r}_{b} r_{c}$ \\
$\tilde{r}_{f}$ & Ice-water interface & $\tilde{r}_{f}=\tilde{r}_{b} r_{f}$ \\
$\tilde{r}_{b}$ & Exterior ice boundary & \\
& & TABLE 1 \\
& Dimensional and dimensionless variable definitions.
\end{tabular}

determining cell damage, and hence optimizing operating conditions, such as the cooling rate. Benson and colleagues have investigated optimal control problems for CPA equilibration, introducing the concept of a CPA toxicity cost function that should be minimized $[6,7,9,13,14]$. We consider a similar toxicity cost function, and add a new cost function to characterize intracellular ice formation, in order to estimate cell damage as a function of cooling rate.

The outline of our paper is as follows. In $\S 2$ we present the full model, nondimensionalize, and provide numerical solutions to illustrate the qualitative behaviours of the system. In $\S 3$ we perform an asymptotic analysis, exploiting the separation of the three natural timescales inherent to the problem. In $\S 4$ we explore how the general analysis of the previous section can be reduced in three distinguished limits of the system where the operationally imposed cooling rate matches each of the natural timescales. In particular, we show when it is important to account for spatial dependence, and when the system can be formally reduced to a system of ODEs similar to the KK model discussed above [26, 29]. We also validate our asymptotic results by comparison with numerical solutions of the full model. In $\S 5$ we introduce damage metrics to account for cell damage due to supercooling (where liquid is cooled to below its freezing point without solidification) and CPA toxicity. We conclude in $\S 6$ with a discussion of our results, and suggestions for further model extensions.

2. Model description. We consider the problem of a cell immersed in a liquid medium containing two chemical species. One of the species, labelled $\tilde{x}$, is able to permeate the cell membrane but the other, $\tilde{y}$, is not. As cryoprotective agents (CPAs) are partly chosen for their ability to permeate the cell, we refer to the permeable species as CPA, and refer to the impermeable species simply as the ion species. We assume that the concentration of both species is initially equal inside and outside the cell, with the entire system at the initial freezing point of the liquid. The system is cooled at the external boundary at a prescribed rate to a final temperature $\tilde{T}_{\text {end }}$, after which we allow the system to equilibrate. The rate of the cooling is a key parameter we investigate. As the system cools, the formation of ice from the external boundary into the interior reduces the volume of the liquid phase, concentrating the chemical species. This changes the freezing point of the liquid phase, and also induces an osmotic pressure across the cell membrane, driving a change in cell volume. We provide a schematic of the dimensionless problem in Figure 1 and a list of variables in Table 1. For brevity, we will not discuss the parameter definitions in the main text, these are all contained in Table 2.

We consider a spherically symmetric domain in which the cell centre is located at the origin, and $\tilde{r}$ is the radial coordinate. The cell domain is $0<\tilde{r}<\tilde{r}_{c}$, where $\tilde{r}=\tilde{r}_{c}(\tilde{t})$ defines the cell membrane position and $\tilde{t}$ denotes time. The fluid domain is $\tilde{r}_{c}<\tilde{r}<\tilde{r}_{f}$, where $\tilde{r}=\tilde{r}_{f}(\tilde{t})$ defines the freezing front position. The ice domain is $\tilde{r}_{f}<\tilde{r}<\tilde{r}_{b}$, where $\tilde{r}=\tilde{r}_{b}$ defines the external boundary. The cell membrane and the freezing front are moving boundaries which must be determined as part of the solution. We note that while conservation of mass should result in a slight expansion of the exterior ice boundary as the water freezes, resulting in a third moving boundary, this effect is small and we therefore neglect it as a simplification. 


\begin{tabular}{|c|c|c|}
\hline Parameters & Typical value & Description \\
\hline$\tilde{D}_{c}^{x}$ & $2 \times 10^{-10} \mathrm{~m}^{2} \mathrm{~s}^{-1}[15]$ & Intracellular CPA diffusivity \\
\hline$\tilde{D}_{c}^{y}$ & $4 \times 10^{-10} \mathrm{~m}^{2} \mathrm{~s}^{-1}[15]$ & Intracellular ion diffusivity \\
\hline$\tilde{D}_{l}^{x}$ & $1 \times 10^{-9} \mathrm{~m}^{2} \mathrm{~s}^{-1}[15]$ & Extracellular CPA diffusivity \\
\hline$\tilde{D}_{l}^{y}$ & $2 \times 10^{-9} \mathrm{~m}^{2} \mathrm{~s}^{-1}[15]$ & Extracellular ion diffusivity \\
\hline$\tilde{k}_{l}{ }_{l}$ & $0.6 \mathrm{~W} \mathrm{~K}^{-1} \mathrm{~m}^{-1}$ & Thermal conductivity of water \\
\hline$\tilde{k}_{s}$ & $2.2 \mathrm{~W} \mathrm{~K}^{-1} \mathrm{~m}^{-1}$ & Thermal conductivity of ice \\
\hline$\tilde{\rho}_{l}$ & $1 \times 10^{3} \mathrm{~kg} \mathrm{~m}^{-3}$ & Density of water \\
\hline$\tilde{\rho}_{s}$ & $9 \times 10^{2} \mathrm{~kg} \mathrm{~m}^{-3}$ & Density of ice \\
\hline$\tilde{c}_{l}$ & $4 \times 10^{3} \mathrm{~J} \mathrm{~kg}^{-1} \mathrm{~K}^{-1}$ & Specific heat capacity of water \\
\hline$\tilde{c}_{s}$ & $2 \times 10^{3} \mathrm{~J} \mathrm{~kg}^{-1} \mathrm{~K}^{-1}$ & Specific heat capacity of ice \\
\hline$\tilde{L}$ & $3.4 \times 10^{5} \mathrm{~J} \mathrm{~kg}^{-1}$ & Latent heat of freezing water \\
\hline$\tilde{\alpha}$ & $4 \times 10^{-3} \mathrm{~K} \mathrm{~m}^{3} \mathrm{~mol}^{-1}[28]$ & Cryoscopic constant of CPA \\
\hline$\tilde{\gamma}$ & $0 \mathrm{~K} \mathrm{~m}^{3} \mathrm{~mol}^{-1}$ & Cryoscopic constant of ion species \\
\hline$\tilde{r}_{c 0}$ & $5 \times 10^{-5} \mathrm{~m}[45]$ & Initial cell radius \\
\hline$\tilde{r}_{b}$ & $5 \times 10^{-4} \mathrm{~m}[32]$ & System radius \\
\hline$\tilde{\kappa}$ & $5 \times 10^{-15} \mathrm{~m}^{2} \mathrm{~s} \mathrm{~kg}^{-1}[15]$ & Hydraulic conductivity of cell membrane \\
\hline$\tilde{\omega}$ & $5 \times 10^{-14} \mathrm{~s} \mathrm{~mol} \mathrm{~m}^{-1} \mathrm{~kg}^{-1}[15]$ & CPA permeability of cell membrane \\
\hline$\tilde{R}$ & $8.3 \mathrm{~kg} \mathrm{~m}^{2} \mathrm{~s}^{-2} \mathrm{~K}^{-1} \mathrm{~mol}^{-1}$ & Universal gas constant \\
\hline$\sigma$ & $0.65[8]$ & CPA reflection coefficient at cell membrane \\
\hline$\tilde{X}_{0}$ & $1 \times 10^{3} \mathrm{~mol} \mathrm{~m}^{-3}[34]$ & Initial CPA concentration (intra and extra) \\
\hline$\tilde{Y}_{0}$ & $1 \times 10^{2} \mathrm{~mol} \mathrm{~m}^{-3}[23]$ & Initial ion concentration (intra and extra) \\
\hline$\tilde{T}_{0}=\tilde{T}_{f 0}-\tilde{\alpha} \tilde{X}_{0}$ & $269 \mathrm{~K}$ & Initial temperature of system \\
\hline$\tilde{T}_{\text {end }}$ & $200 \mathrm{~K}[42]$ & Final temperature of system \\
\hline$\tilde{T}_{f 0}$ & $273 \mathrm{~K}$ & Freezing temperature of water in absence of CPA \\
\hline$\tilde{\beta}$ & $10^{-3}-10^{5} \mathrm{~K} \mathrm{~s}^{-1}[38,40]$ & Cooling rate of exterior boundary \\
\hline
\end{tabular}

Parameters from the full dimensional problem and their typical values. We estimate $\tilde{D}_{c}^{x}$ using the observation in Verkman [47] that small molecules have a diffusivity of around five times less in cytoplasm than in water, and we estimate $\tilde{D}_{c}^{y}$ and $\tilde{D}_{l}^{y}$ by the observation that ions tend to be much smaller molecules than CPA agents, and so ion diffusivity will be higher in a given medium. We use the typical cell radius of the human oocyte.

We track the temperature in the cell and liquid, $\tilde{T}_{l}$, and the ice, $\tilde{T}_{s}$. We assume that heat conduction in the cell is the same as that in the liquid as the cell consists mainly of water, so we do not differentiate between these two regions for the heat flow problem. We also track the concentrations of the cryoprotective agent $(\mathrm{CPA})$ and the ion species in the cell $\left(\tilde{x}_{c}, \tilde{y}_{c}\right)$ and liquid $\left(\tilde{x}_{l}, \tilde{y}_{l}\right)$. As discussed above, the freezing point of the liquid will be lowered by the presence of chemical species, and we define the freezing temperature $\tilde{T}_{f}$ as

$$
\tilde{T}_{f}(\tilde{x}, \tilde{y}):=\tilde{T}_{f 0}-\tilde{\alpha} \tilde{x}-\tilde{\gamma} \tilde{y}
$$

where we assume a linear relationship. For simplicity, we will consider the case where the freezing temperature depends on the CPA concentration only, so we take $\tilde{\gamma}=0 \mathrm{~K} \mathrm{~m}^{3} \mathrm{~mol}^{-1}$. This means that the role of the ion species in this model is to impart an osmotic pressure across the cell membrane as the ice region grows and the extracellular liquid volume is decreased, resulting in a change in cell volume. The analysis could easily be extended to incorporate the dependence of the freezing temperature on the ion concentration.

We assume that the chemical transport in the cell and liquid is due to diffusion. While we expect the chemical diffusivity and membrane parameters to depend on temperature in practice [15], we treat these parameters as independent of temperature for simplicity and to facilitate analytical progress. We provide justification of this assumption in Appendix A, where we show that the qualitative features of the problem do not change significantly when temperature dependence is taken into account. 
The equations governing the temperature distribution in the liquid and ice are

$$
\begin{array}{cc}
\tilde{\rho}_{l} \tilde{c}_{l} \frac{\partial \tilde{T}_{l}}{\partial \tilde{t}}=\frac{\tilde{k}_{l}}{\tilde{r}^{2}} \frac{\partial}{\partial \tilde{r}}\left(\tilde{r}^{2} \frac{\partial \tilde{T}_{l}}{\partial \tilde{r}}\right) \quad \text { for } 0<\tilde{r}<\tilde{r}_{f}(\tilde{t}), \\
\tilde{\rho}_{s} \tilde{c}_{s} \frac{\partial \tilde{T}_{s}}{\partial \tilde{t}}=\frac{\tilde{k}_{s}}{\tilde{r}^{2}} \frac{\partial}{\partial \tilde{r}}\left(\tilde{r}^{2} \frac{\partial \tilde{T}_{s}}{\partial \tilde{r}}\right) & \text { for } \tilde{r}_{f}(\tilde{t})<\tilde{r}<\tilde{r}_{b},
\end{array}
$$

and those governing solute diffusion are

$$
\begin{array}{r}
\frac{\partial \tilde{x}_{c}}{\partial \tilde{t}}=\frac{\tilde{D}_{c}^{x}}{\tilde{r}^{2}} \frac{\partial}{\partial \tilde{r}}\left(\tilde{r}^{2} \frac{\partial \tilde{x}_{c}}{\partial \tilde{r}}\right) \quad \text { for } 0<\tilde{r}<\tilde{r}_{c}(\tilde{t}), \\
\frac{\partial \tilde{y}_{c}}{\partial \tilde{t}}=\frac{\tilde{D}_{c}^{y}}{\tilde{r}^{2}} \frac{\partial}{\partial \tilde{r}}\left(\tilde{r}^{2} \frac{\partial \tilde{y}_{c}}{\partial \tilde{r}}\right) \quad \text { for } 0<\tilde{r}<\tilde{r}_{c}(\tilde{t}), \\
\frac{\partial \tilde{x}_{l}}{\partial \tilde{t}}=\frac{\tilde{D}_{l}^{x}}{\tilde{r}^{2}} \frac{\partial}{\partial \tilde{r}}\left(\tilde{r}^{2} \frac{\partial \tilde{x}_{l}}{\partial \tilde{r}}\right) \quad \text { for } \tilde{r}_{c}(\tilde{t})<\tilde{r}<\tilde{r}_{f}(\tilde{t}), \\
\frac{\partial \tilde{y}_{l}}{\partial \tilde{t}}=\frac{\tilde{D}_{l}^{y}}{\tilde{r}^{2}} \frac{\partial}{\partial \tilde{r}}\left(\tilde{r}^{2} \frac{\partial \tilde{y}_{l}}{\partial \tilde{r}}\right) \quad \text { for } \tilde{r}_{c}(\tilde{t})<\tilde{r}<\tilde{r}_{f}(\tilde{t}) .
\end{array}
$$

We now consider appropriate boundary and coupling conditions. At the origin, we impose symmetry conditions

$$
\tilde{D}_{c}^{x} \frac{\partial \tilde{x}_{c}}{\partial \tilde{r}}=0, \quad \tilde{D}_{c}^{y} \frac{\partial \tilde{y}_{c}}{\partial \tilde{r}}=0, \quad \tilde{k}_{l} \frac{\partial \tilde{T}_{l}}{\partial \tilde{r}}=0, \quad \text { for } \tilde{r}=0 .
$$

At the cell membrane, we impose the following conservation conditions:

$$
\begin{array}{rr}
\tilde{D}_{c}^{x} \frac{\partial \tilde{x}_{c}}{\partial \tilde{r}}+\tilde{x}_{c} \frac{\mathrm{d} \tilde{r}_{c}}{\mathrm{~d} \tilde{t}}=\tilde{D}_{l}^{x} \frac{\partial \tilde{x}_{l}}{\partial \tilde{r}}+\tilde{x}_{l} \frac{\mathrm{d} \tilde{r}_{c}}{\mathrm{~d} \tilde{t}}=\tilde{\omega} \tilde{R} \tilde{T}_{0}\left(\tilde{x}_{l}-\tilde{x}_{c}\right) & \text { for } \tilde{r}=\tilde{r}_{c}(\tilde{t}), \\
\tilde{D}_{c}^{y} \frac{\partial \tilde{y}_{c}}{\partial \tilde{r}}+\tilde{y}_{c} \frac{\mathrm{d} \tilde{r}_{c}}{\mathrm{~d} \tilde{t}}=\tilde{D}_{l}^{y} \frac{\partial \tilde{y}_{l}}{\partial \tilde{r}}+\tilde{y}_{l} \frac{\mathrm{d} \tilde{r}_{c}}{\mathrm{~d} \tilde{t}}=0 & \text { for } \tilde{r}=\tilde{r}_{c}(\tilde{t}), \\
\frac{\mathrm{d} \tilde{r}_{c}}{\mathrm{~d} \tilde{t}}=-\tilde{\kappa} \tilde{R} \tilde{T}_{0}\left[\sigma\left(\tilde{x}_{l}-\tilde{x}_{c}\right)+\left(\tilde{y}_{l}-\tilde{y}_{c}\right)\right] & \text { for } \tilde{r}=\tilde{r}_{c}(\tilde{t}) .
\end{array}
$$

Equation (5a) corresponds to continuity of CPA flux through the cell membrane, and that this flux is proportional to the concentration difference across the membrane. Equation (5b) corresponds to no ion flux across the membrane. Equation (5c) corresponds to the dynamic change in cell volume being proportional to the osmotic pressure difference across the cell surface. We use a reflection coefficient of 1 for the difference in ion concentration in (5c) since we assume that the cell membrane is impermeable to ions [29]. Additionally, we have neglected surface tension effects, as a simple dimensional analysis shows that typical surface tension values of around $0.01-0.1 \mathrm{mN} \mathrm{m}^{-1}$ for the animal cell membrane [11,48] yield effects approximately six orders of magnitude weaker than osmotic forces.

At the freezing front, we have the following conditions:

$$
\begin{array}{rr}
\tilde{T}_{l}=\tilde{T}_{s}=\tilde{T}_{f}\left(\tilde{x}_{l}\right) & \text { for } \tilde{r}=\tilde{r}_{f}(\tilde{t}), \\
\tilde{D}_{l}^{x} \frac{\partial \tilde{x}_{l}}{\partial \tilde{r}}+\tilde{x}_{l} \frac{\mathrm{d} \tilde{r}_{f}}{\mathrm{~d} \tilde{t}}=0 & \text { for } \tilde{r}=\tilde{r}_{f}(\tilde{t}), \\
\tilde{D}_{l}^{y} \frac{\partial \tilde{y}_{l}}{\partial \tilde{r}}+\tilde{y}_{l} \frac{\mathrm{d} \tilde{r}_{f}}{\mathrm{~d} \tilde{t}}=0 & \text { for } \tilde{r}=\tilde{r}_{f}(\tilde{t}), \\
\tilde{\rho}_{s} \tilde{L} \frac{\mathrm{d} \tilde{r}_{f}}{\mathrm{~d} \tilde{t}}=\tilde{k}_{s} \frac{\partial \tilde{T}_{s}}{\partial \tilde{r}}-\tilde{k}_{l} \frac{\partial \tilde{T}_{l}}{\partial \tilde{r}} & \text { for } \tilde{r}=\tilde{r}_{f}(\tilde{t}) .
\end{array}
$$

Here, (6a) corresponds to the temperature at the freezing front being continuous and depending on the CPA concentration as defined in (1), (6b) and (6c) ensure no flux of either solute across the freezing front, and 


\begin{tabular}{ll} 
Dimensionless parameter & Typical value \\
\hline$k_{l}=\tilde{k}_{l} \tilde{\rho}_{s} \tilde{c}_{s} /\left(\tilde{k}_{s} \tilde{\rho}_{l} \tilde{c}_{l}\right)$ & $1.2 \times 10^{-1}$ \\
$k=\tilde{k}_{l} / \tilde{k}_{s}$ & $2.7 \times 10^{-1}$ \\
$S=\tilde{L} /\left(\tilde{c}_{s}\left(\tilde{T}_{f 0}-\tilde{T}_{\text {end }}\right)\right)$ & $2.3 \times 10^{0}$ \\
$D_{c}^{x}=\tilde{D}_{c}^{x} \tilde{\rho}_{s} \tilde{c}_{s} / \tilde{k}_{s}$ & $1.6 \times 10^{-4}$ \\
$D_{c}^{y}=\tilde{D}_{c}^{y} \tilde{\rho}_{s} \tilde{c}_{s} / \tilde{k}_{s}$ & $3.3 \times 10^{-4}$ \\
$D_{l}^{x}=\tilde{D}_{l}^{x} \tilde{\rho}_{s} \tilde{c}_{s} / \tilde{k}_{s}$ & $8.2 \times 10^{-4}$ \\
$D_{l}^{y}=\tilde{D}_{l}^{y} \tilde{\rho}_{s} \tilde{c}_{s} / \tilde{k}_{s}$ & $1.6 \times 10^{-3}$ \\
$\kappa=\tilde{\kappa} \tilde{R} \tilde{T}_{0} \tilde{X}_{0} \tilde{\rho}_{s} \tilde{c}_{s} \tilde{r}_{b} / \tilde{k}_{s}$ & $4.6 \times 10^{-6}$ \\
$\omega=\tilde{\omega} \tilde{T}_{0} \tilde{\rho}_{s} \tilde{c}_{s} \tilde{r}_{b} / \tilde{k}_{s}$ & $4.6 \times 10^{-8}$ \\
$\alpha=\tilde{\alpha} \tilde{X}_{0} /\left(\tilde{T}_{f 0}-\tilde{T}_{\text {end }}\right)$ & $5.5 \times 10^{-2}$ \\
$\beta=\tilde{\beta} \tilde{\rho}_{s} \tilde{c}_{s} \tilde{r}_{b}^{2} /\left(\tilde{k}_{s}\left(\tilde{T}_{f 0}-\tilde{T}_{\text {end }}\right)\right)$ & $2.8 \times 10^{-6}-2.8 \times 10^{2}$ \\
$r_{c 0}=\tilde{r}_{c 0} / \tilde{r}_{b}$ & $1 \times 10^{-1}$ \\
$Y_{0}=\tilde{Y}_{0} / \tilde{X}_{0}$ & $1 \times 10^{-1}$
\end{tabular}

Dimensionless parameters, with typical values derived from Table 2. Note that $\sigma$ is provided in Table 2.

(6d) corresponds to the Stefan condition, namely that latent heat is released as water is frozen, which results in the velocity of the interface being proportional to the difference in heat flux across the interface [19].

Finally, at the exterior ice boundary, we prescribe the temperature in terms of a general function $\tilde{T}_{p}(\tilde{t})$ :

$$
\tilde{T}_{s}=\tilde{T}_{p}(\tilde{t}) \quad \text { for } \tilde{r}=\tilde{r}_{b} .
$$

The initial conditions of the system are as follows

$$
\begin{array}{r}
\tilde{x}_{c}(\tilde{r}, 0)=\tilde{X}_{0}, \quad \tilde{x}_{l}(\tilde{r}, 0)=\tilde{X}_{0}, \quad \tilde{y}_{c}(\tilde{r}, 0)=\tilde{Y}_{0}, \quad \tilde{y}_{l}(\tilde{r}, 0)=\tilde{Y}_{0}, \\
\tilde{T}_{l}(\tilde{r}, 0)=\tilde{T}_{0}:=\tilde{T}_{f 0}-\tilde{\alpha} \tilde{X}_{0}, \quad \tilde{r}_{c}(0)=\tilde{r}_{c 0}, \quad \tilde{r}_{f}(0)=\tilde{r}_{b} .
\end{array}
$$

corresponding to a system where the CPA and ion concentration are the same within the cell and the liquid, and the entire system is at the initial freezing temperature of the liquid. The prescribed final temperature is $\tilde{T}_{\text {end }}$, so the prescribed temperature change will be $\tilde{T}_{f 0}-\tilde{\alpha} \tilde{X}_{0}-\tilde{T}_{\text {end }}$. For later use, we introduce a typical cooling rate $\tilde{\beta}$ (which can be experimentally controlled) and hence have a timescale for cooling, $\left(\tilde{T}_{f 0}-\tilde{\alpha} \tilde{X}_{0}-\tilde{T}_{\text {end }}\right) / \tilde{\beta}$.

Taken together, our system consists of equations (2)-(3), boundary conditions (4)-(7), and initial conditions (8).

2.1. Dimensionless problem. We form the dimensionless variables specified in Table 1 and the dimensionless parameters specified in Table 3. We choose our time scaling according to the fastest timescale in the problem, thermal conduction, which occurs over a few seconds in dimensional time.

Using the nondimensionalization described above, the thermal diffusion problems (2) become

$$
\begin{array}{cl}
\frac{\partial T_{l}}{\partial t}=\frac{k_{l}}{r^{2}} \frac{\partial}{\partial r}\left(r^{2} \frac{\partial T_{l}}{\partial r}\right) & \text { for } 0<r<r_{f}(t), \\
\frac{\partial T_{s}}{\partial t}=\frac{1}{r^{2}} \frac{\partial}{\partial r}\left(r^{2} \frac{\partial T_{s}}{\partial r}\right) & \text { for } r_{f}(t)<r<1,
\end{array}
$$

and the solute diffusion problems (3) become

$$
\begin{array}{ll}
\frac{\partial x_{c}}{\partial t}=\frac{D_{c}^{x}}{r^{2}} \frac{\partial}{\partial r}\left(r^{2} \frac{\partial x_{c}}{\partial r}\right) & \text { for } 0<r<r_{c}(t), \\
\frac{\partial y_{c}}{\partial t}=\frac{D_{c}^{y}}{r^{2}} \frac{\partial}{\partial r}\left(r^{2} \frac{\partial y_{c}}{\partial r}\right) & \text { for } 0<r<r_{c}(t),
\end{array}
$$




$$
\begin{array}{ll}
\frac{\partial x_{l}}{\partial t}=\frac{D_{l}^{x}}{r^{2}} \frac{\partial}{\partial r}\left(r^{2} \frac{\partial x_{l}}{\partial r}\right) & \text { for } r_{c}(t)<r<r_{f}(t), \\
\frac{\partial y_{l}}{\partial t}=\frac{D_{l}^{y}}{r^{2}} \frac{\partial}{\partial r}\left(r^{2} \frac{\partial y_{l}}{\partial r}\right) & \text { for } r_{c}(t)<r<r_{f}(t) .
\end{array}
$$

At the origin, the symmetry conditions (4) become

$$
D_{c}^{x} \frac{\partial x_{c}}{\partial r}=0, \quad D_{c}^{y} \frac{\partial y_{c}}{\partial r}=0, \quad k_{l} \frac{\partial T_{l}}{\partial r}=0, \quad \text { for } r=0 .
$$

At the cell membrane, the boundary conditions (5) become

$$
\begin{array}{rr}
D_{c}^{x} \frac{\partial x_{c}}{\partial r}+x_{c} \frac{\mathrm{d} r_{c}}{\mathrm{~d} t}=D_{l}^{x} \frac{\partial x_{l}}{\partial r}+x_{l} \frac{\mathrm{d} r_{c}}{\mathrm{~d} t}=\omega\left(x_{l}-x_{c}\right) & \text { for } r=r_{c}(t), \\
D_{c}^{y} \frac{\partial y_{c}}{\partial r}+y_{c} \frac{\mathrm{d} r_{c}}{\mathrm{~d} t}=D_{l}^{y} \frac{\partial y_{l}}{\partial r}+y_{l} \frac{\mathrm{d} r_{c}}{\mathrm{~d} t}=0 & \text { for } r=r_{c}(t), \\
\frac{\mathrm{d} r_{c}}{\mathrm{~d} t}=-\kappa\left[\sigma\left(x_{l}-x_{c}\right)+\left(y_{l}-y_{c}\right)\right] & \text { for } r=r_{c}(t) .
\end{array}
$$

At the freezing front, the boundary conditions (6) become

$$
\begin{aligned}
T_{l}=T_{s}=T_{f} & \text { for } r=r_{f}(t), \\
D_{l}^{x} \frac{\partial x_{l}}{\partial r}+x_{l} \frac{\mathrm{d} r_{f}}{\mathrm{~d} t}=0 & \text { for } r=r_{f}(t), \\
D_{l}^{y} \frac{\partial y_{l}}{\partial r}+y_{l} \frac{\mathrm{d} r_{f}}{\mathrm{~d} t}=0 & \text { for } r=r_{f}(t), \\
S \frac{\mathrm{d} r_{f}}{\mathrm{~d} t}=\frac{\partial T_{s}}{\partial r}-k \frac{\partial T_{l}}{\partial r} & \text { for } r=r_{f}(t),
\end{aligned}
$$

where the dimensionless freezing temperature $T_{f}$ is

$$
T_{f}\left(x_{l}\right):=-\alpha x_{l} .
$$

At the exterior ice boundary, the boundary condition (7) becomes

$$
T_{s}=T_{p}(t),
$$

where $T_{p}(0)=-\alpha$. The dimensionless cooling rate is denoted $\beta$, and the final temperature is $T_{p}=-1$. We will mainly consider linear cooling in the numerics, so

$$
T_{p}(t)= \begin{cases}-\alpha-\beta t & \text { for } 0<t<(1-\alpha) / \beta, \\ -1 & \text { for } t>(1-\alpha) / \beta\end{cases}
$$

Finally, from (8), the initial conditions of the system are now

$$
(17) x_{c}(r, 0)=1, \quad x_{l}(r, 0)=1, \quad y_{c}(r, 0)=Y_{0}, \quad y_{l}(r, 0)=Y_{0}, \quad T_{l}(r, 0)=-\alpha, \quad r_{c}(0)=r_{c 0}, \quad r_{f}(0)=1 .
$$

Our dimensionless system is thus defined by equations (9)-(10), with boundary conditions (11)-(15) and initial conditions (17). We now solve this system numerically to understand the qualitative behaviour, before undertaking an asymptotic analysis in $§ 3$.

2.2. Numerical solutions. We solve this system numerically via the following steps. First, we move to new dependent variables of the form $r f(r, t)$, where $f$ represents any of the dependent variables in the system. This reduces the governing equations to one-dimensional diffusion in Cartesian coordinates. Then we impose the Landau transformation [30] to fix the moving boundary in each phase. We implement the method of lines with a discretization in space (focused near boundary layers if necessary), essentially converting our partial differential equations into a system of ordinary differential equations. We solve this system in time using the function ode15s in MATLAB. This procedure is outlined in more detail in Appendix B. One issue 

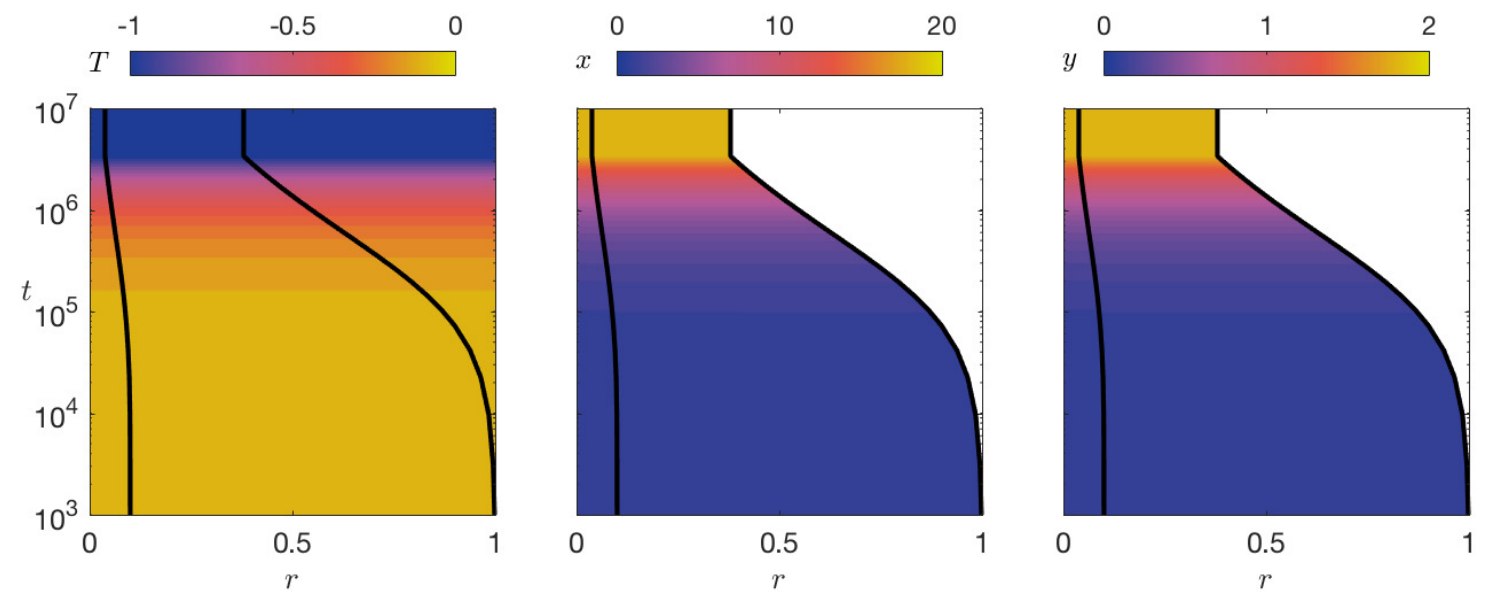

FIG. 2. Numerical solutions for a slow dimensionless cooling rate $\left(\beta=2.8 \times 10^{-7}\right.$, corresponding to a dimensional cooling rate of $\left.\tilde{\beta}=10^{-4} \mathrm{~K} \mathrm{~s}^{-1}\right)$. From left to right, we show the temperature, CPA concentration, and ion concentration. The black lines represent the position of the cell membrane and the freezing front, respectively. All processes occur over the osmotic timescale here, roughly corresponding to $t>1000$.

we face is that the ice region does not exist at $t=0$. To circumvent this, we start our simulations at a small but finite time, using initial conditions that are consistent with the asymptotic early-time behaviour (see Appendix C).

To explore different cooling rates via our numerical scheme, we use the functional form in (16), and interrogate the solution with different choices of cooling rate $\beta$. We emphasize that the asymptotic analysis presented in $\S 3$ is valid for a more general class of prescribed temperatures on the external boundary, including this simple case of linear cooling. It is the cooling rate that is important to the asymptotic analysis, not the exact form of the prescribed temperature.

The system behaviour changes significantly as the cooling rate varies. The variables are approximately spatially homogeneous for lower cooling rates (Figure 2), but develop spatial gradients as the cooling rate increases: first occurring for the concentration (Figure 3), then also for the temperature (Figure 4) if the cooling rate is very rapid. While the final equilibrium state is the same for all cooling rates, the dynamics of how these states are reached differ with the cooling rate.

The spatial gradients in solute concentrations in Figures 3-4 are restricted to the liquid phase; the solute concentrations within the cell always appear to be spatially homogeneous (Figures 2-4). While the change in solute concentration and temperature at the cell centre occur over the same timescale when the cooling rate is low (Figure 2), the change in solute concentration occurs over a slower timescale than for the temperature when the cooling rate is faster (Figures 3-4).

The positions of the moving boundaries are highly dependent on the cooling rate (Figures 5-6). The cell membrane and freezing front reach a limiting behaviour in time as the cooling rate increases (Figures $5 \mathrm{a}$ and $6 \mathrm{a}$ ). In addition, the positions of the moving boundaries also tend towards limiting behaviours as the cooling rate decreases. This is most evident when scaled against the external temperature (Figures 5b and $6 \mathrm{~b})$, and it represents quasi-steady kinetics. These observations will be elucidated through our asymptotic analysis in $\S 3$.

\section{General asymptotic analysis.}

3.1. Asymptotic structure. Table 3 shows that there are several small parameters in the system, and that the difference in magnitude between these parameters can be extreme. In this section we use asymptotic techniques to investigate the impact of these differences on the system dynamics.

We identify three natural timescales, associated with thermal conduction $(t=O(1)$, given the nondimensionalization), solute diffusion $\left(t=O\left(1 / D_{l}^{x}\right)\right)$, and osmosis $(t=O(1 / \kappa))$. The relative sizes of these 

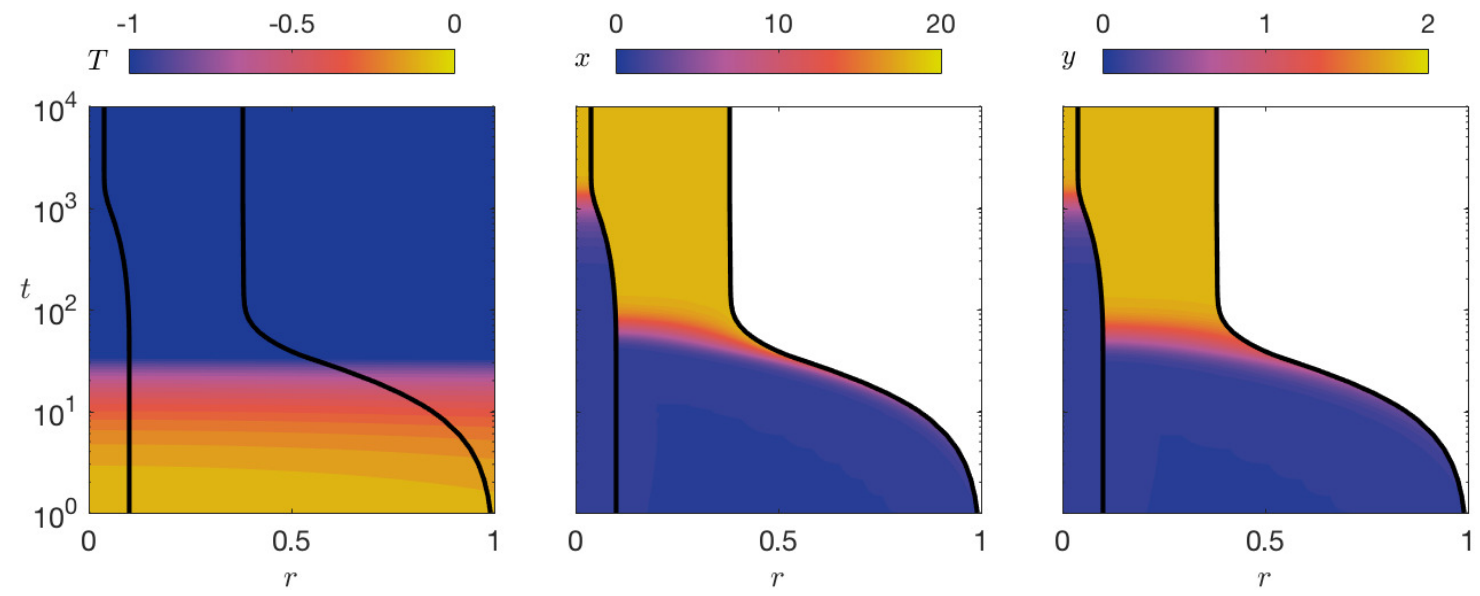

FIG. 3. Numerical solutions for a moderate dimensionless cooling rate $\left(\beta=2.8 \times 10^{-2}\right.$, corresponding to a dimensional cooling rate of $\tilde{\beta}=10^{1} \mathrm{~K} \mathrm{~s}^{-1}$ ). As in Figure 2, from left to right we show the temperature, CPA concentration, and ion concentration. The black lines represent the position of the cell membrane and the freezing front, respectively. The solute diffusion timescale roughly corresponds to $20<t<200$, and the osmotic timescale to $200<t<3000$.
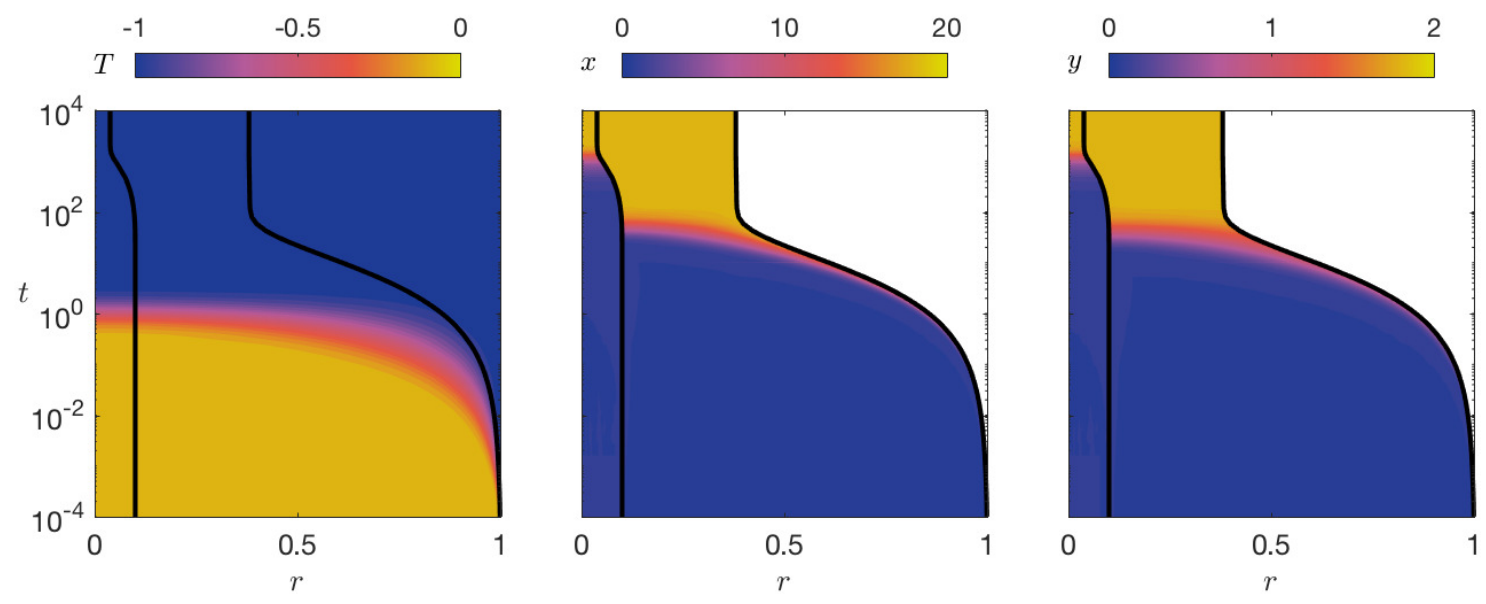

FIG. 4. Numerical solutions for a rapid dimensionless cooling rate $\left(\beta=2.8 \times 10^{3}\right.$, corresponding to a dimensional cooling rate of $\tilde{\beta}=10^{6} \mathrm{~K} \mathrm{~s}^{-1}$ ). As in Figure 2, from left to right we show the temperature, CPA concentration, and ion concentration. The black lines represent the position of the cell membrane and the freezing front, respectively. The thermal conduction timescale roughly corresponds to $t<10$, the solute diffusion timescale to $10<t<200$, and the osmotic timescale to $200<t<3000$.

timescales are summarized in Figure 7. The imposed cooling timescale $1 / \beta$ may overlap with any of these, as discussed in $\S 4$. To perform the asymptotic analysis, we assume the limit $\kappa \ll D_{l}^{x} \ll 1$ henceforth, so that the timescales outlined above are well separated. When we refer to 'leading-order' in the next sections, this is meant in the asymptotic limit $0<\kappa<D_{l}^{x} \rightarrow 0$. We are motivated by the values in Table 3 to treat $D_{c}^{x}, D_{c}^{y}, D_{l}^{y}$ all as the same (small) order as $D_{l}^{x}$, and to treat $\omega$ as the same (even smaller) order as $\kappa$. All other parameters $\left(k_{l}, k, S, \alpha, r_{c 0}, Y_{0}\right)$ are treated as $O(1)$. In the next three subsections $(\S 3.2-\S 3.4)$ we discuss each timescale in turn, from fastest to slowest. We start each subsection by summarising the physical implications of the results we will deduce in that subsection. 

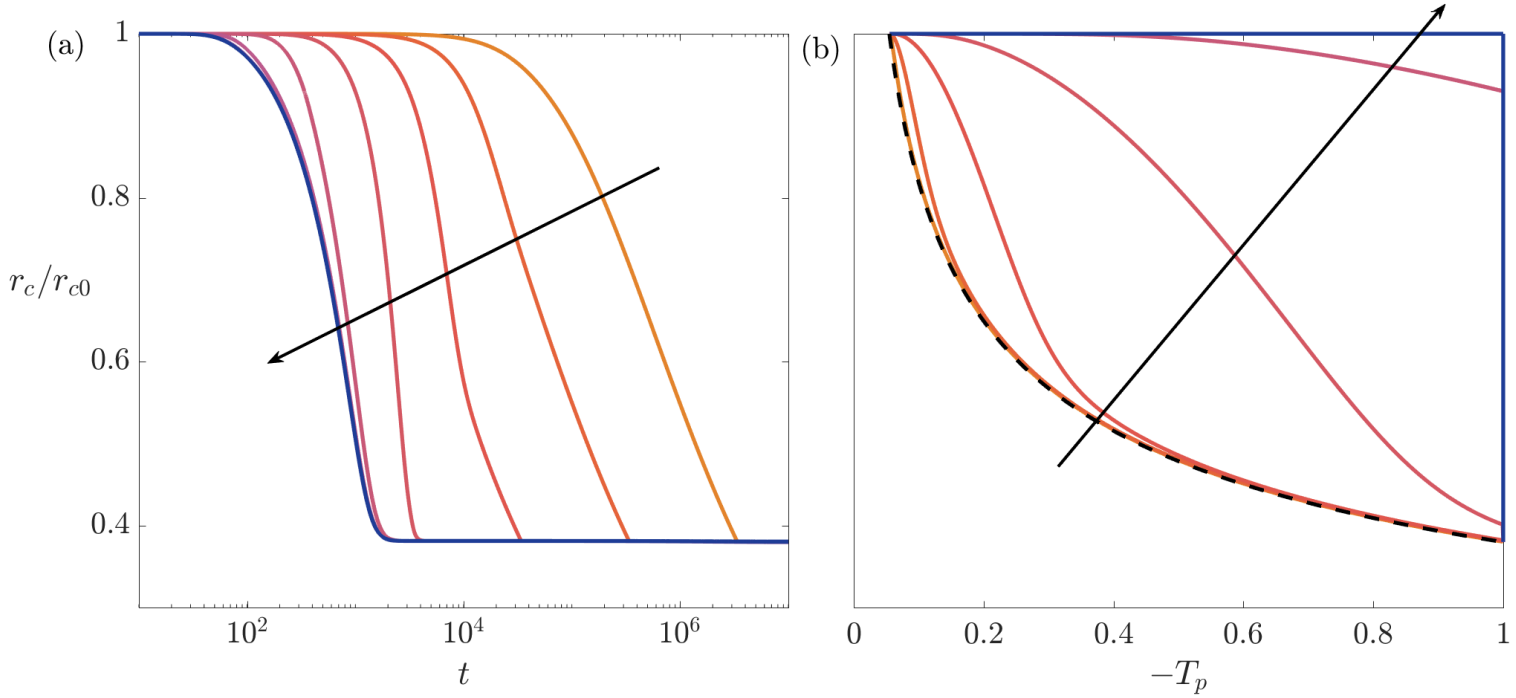

FIG. 5. The normalized radius of the cell $r_{c}(t) / r_{c 0}$ during freezing, plotted against (a) time and (b) prescribed temperature $T_{p}(t)$ at the external boundary. The different solid lines correspond to different values of the cooling rate; we use $\beta=2.8 \times 10^{k}$, where $k=\{-7, \ldots, 3\}$ (corresponding to dimensional cooling rates ranging between $10^{-4}$ and $10^{6} \mathrm{~K} \mathrm{~s}^{-1}$ ). Otherwise, we use the parameter values in Table 3. The dashed line is the quasi-steady solution from (44). The arrows denote increasing cooling rate.

3.2. Thermal conduction timescale: $t=O(1)$. Over this timescale, the important process is heat conduction, and there can be a significant spatial temperature variation. The moving boundaries are static to leading order, and the chemical concentrations remain unchanged from their initial values except in a boundary layer near the freezing front, as seen in Figures 3-4. We see in $\S 3.3$ that this boundary layer problem is the early-time version of a broader diffusion problem that occurs at the later timescale. We will therefore relegate the details of the chemical boundary layer to Appendix D.

To formalize the above statements, we note that the cell membrane velocity $\mathrm{d} r_{c} / \mathrm{d} t=O(\kappa)$ and, as seen in Appendix D, the freezing front movement $\mathrm{d} r_{f} / \mathrm{d} t=O\left(\sqrt{D_{l}^{x}}\right)$, resulting in $r_{c}(t)=r_{c 0}$ and $r_{f}(t)=1$ at leading order. Therefore, there is no ice phase at leading order. From (10), the dynamics of $x_{c}$ and $y_{c}$ are unimportant over this timescale; these concentrations remain unchanged from their initial values, as can be seen in Figures 2-4, resulting in $x_{c}=1$ and $y_{c}=Y_{0}$ to leading order.

The only variable that evolves significantly on this timescale is the liquid temperature, $T_{l}$, which satisfies the reduced problem

$$
\frac{\partial T_{l}}{\partial t}=\frac{k_{l}}{r^{2}} \frac{\partial}{\partial r}\left(r^{2} \frac{\partial T_{l}}{\partial r}\right) \quad \text { for } 0<r<1
$$

with symmetry condition

$$
k_{l} \frac{\partial T_{l}}{\partial r}=0 \quad \text { for } r=0
$$

Since the ice region is very small and the temperature within the ice is essentially uniform, we have $T_{s}=T_{p}(t)$ at leading-order (see Appendix D). This leads to the reduced boundary condition

$$
T_{l}=T_{p}(t) \quad \text { for } r=1
$$

Finally, the initial condition is

$$
T_{l}(r, 0)=-\alpha .
$$



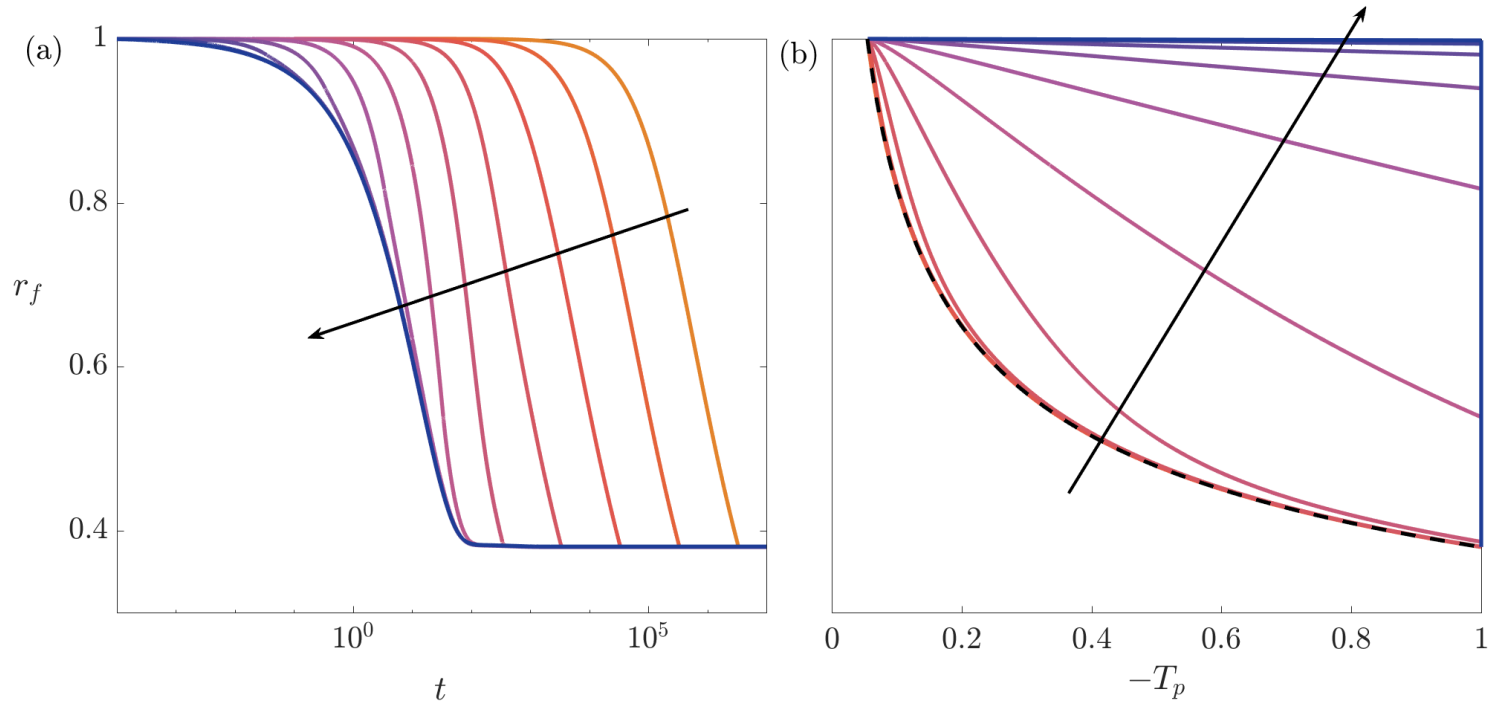

FIG. 6. The radius of the water-ice boundary $r_{f}(t)$ during freezing, plotted against (a) time and (b) prescribed temperature $T_{p}(t)$ at the external boundary. As in Figure 5, the different solid lines correspond to different values of the cooling rate; we use $\beta=2.8 \times 10^{k}$, where $k=\{-7, \ldots, 3\}$ (corresponding to dimensional cooling rates ranging between $10^{-4}$ and $10^{6} \mathrm{~K} \mathrm{~s}^{-1}$ ). Otherwise, we use the parameter values in Table 3. The dashed line is the quasi-steady solution from (44). The arrows denote increasing cooling rate.

$$
\begin{aligned}
& t=O\left(1 / D_{l}^{x}\right) \\
& \tilde{t}=O\left(\tilde{r}_{b}^{2} / \tilde{D}_{l}^{x}\right) \\
& \begin{array}{c}
t=O(1) \\
\tilde{t}=O\left(\tilde{\rho}_{l} \tilde{c}_{l} \tilde{r}_{b}^{2} / \tilde{k}_{l}\right)
\end{array} \\
& \text { CPA/ion diffusivity } \\
& \text { Heat conduction } \\
& \tilde{t}=O\left(\tilde{r}_{c} /\left(\tilde{\kappa} \tilde{R} \tilde{T}_{0} \tilde{X}_{0}\right)\right)
\end{aligned}
$$

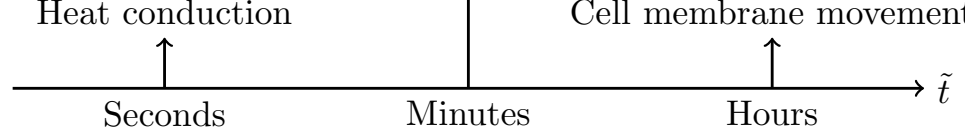

FIG. 7. A schematic of the three natural timescales in the system. The prescribed timescale of cooling is $\tilde{t}=O(1 / \tilde{\beta})$ $(t=O(1 / \beta))$, which will range from seconds to hours.

The reduced temperature problem presented above contains no information about the velocity of the freezing front. In Appendix D, we show that on this timescale the temperature problem is decoupled from the initial motion of the freezing front, the latter being governed by the CPA concentration near the front.

The reduced system for the liquid temperature (18)-(21) has the following analytic leading-order solution

$$
T_{l}=T_{p}(t)+\frac{2}{r} \sum_{n=1}^{\infty} \frac{(-1)^{n} \exp \left(-k_{l} n^{2} \pi^{2} t\right)}{n \pi}\left[\int_{0}^{t} T_{p}^{\prime}(s) \exp \left(k_{l} n^{2} \pi^{2} s\right) \mathrm{d} s\right] \sin n \pi r .
$$

For many prescribed external temperatures (for example, piecewise linear), we can calculate the integral in (22) explicitly.

The large-time behaviour of the temperature over this timescale is given by

$$
T_{l} \rightarrow T_{p}(t), \quad \text { as } t \rightarrow \infty,
$$

and so the spatial dependence of temperature vanishes over this timescale. 
3.3. Solute diffusion timescale: $t=O\left(1 / D_{l}^{x}\right)$. Over this timescale, the important process is solute diffusion within the liquid, and there are spatial variations in concentration. The solutes within the cell are still unchanged from their initial values at leading order, and the temperature is now spatially uniform through the entire system. The cell membrane is static and there is no solute transport through this boundary to leading order. However, the decrease in temperature will cause the freezing front to advance into the liquid. This motion is limited by the concentrating of CPA causing a reduction in the freezing temperature, and by how quickly CPA can be transported away from the front. This is the interesting behaviour on which we focus in this section.

To formalize the above, we introduce the timescale $\tau=D_{l}^{x} t=O(1)$, over which the cell membrane velocity $\mathrm{d} r_{c} / \mathrm{d} \tau=O\left(\kappa / D_{l}^{x}\right)$ is still small, so $r_{c}(\tau)=r_{c 0}$ at leading order. However, the freezing front movement is important, with $\mathrm{d} r_{f} / \mathrm{d} \tau=O(1)$. Over this timescale the thermal conduction is very quick, and it can be shown that the temperature of the entire system is prescribed by the external boundary, that is $T_{s}=T_{l}=T_{p}(\tau)$. From (10), the dynamics of $x_{c}$ and $y_{c}$ are unchanged from their initial conditions over this timescale, with $x_{c}=1$ and $y_{c}=Y_{0}$ at leading order.

The important leading-order system is given by the solute diffusion problem

$$
\frac{\partial x_{l}}{\partial \tau}=\frac{1}{r^{2}} \frac{\partial}{\partial r}\left(r^{2} \frac{\partial x_{l}}{\partial r}\right), \quad \frac{\partial y_{l}}{\partial \tau}=\frac{D_{l}^{y}}{D_{l}^{x} r^{2}} \frac{\partial}{\partial r}\left(r^{2} \frac{\partial y_{l}}{\partial r}\right) \quad \text { for } r_{c 0}<r<r_{f}(\tau),
$$

with the following conditions at the cell membrane

$$
\frac{\partial x_{l}}{\partial r}=0, \quad \frac{\partial y_{l}}{\partial r}=0 \quad \text { for } r=r_{c 0},
$$

and the freezing front

$$
\begin{aligned}
x_{l}=-\frac{T_{p}(\tau)}{\alpha} & \text { for } r=r_{f}(\tau), \\
\frac{\partial x_{l}}{\partial r}+x_{l} \frac{\mathrm{d} r_{f}}{\mathrm{~d} \tau}=0 & \text { for } r=r_{f}(\tau), \\
\frac{D_{l}^{y}}{D_{l}^{x}} \frac{\partial y_{l}}{\partial r}+y_{l} \frac{\mathrm{d} r_{f}}{\mathrm{~d} \tau}=0 & \text { for } r=r_{f}(\tau) .
\end{aligned}
$$

The matching conditions for this system into the earlier timescale as $\tau \rightarrow 0$ are as follows

$$
x_{l}(r, 0)=1, \quad y_{l}(r, 0)=Y_{0}, \quad r_{f}(0)=1 .
$$

We note that these 'initial' conditions may be inconsistent with the boundary conditions at the freezing front (26). This discrepancy is remedied by the early-time boundary layer near the freezing front (over the timescale $t=O(1))$ discussed in the previous section and outlined in Appendix D.

Thus, the problem reduces to that of solute diffusion in the liquid domain, with one moving boundary with a prescribed solute concentration and no flux through either boundary. Moreover, we note that the freezing front movement is limited by how quickly CPA can diffuse away from the interface over this timescale; the problem for the ion concentration decouples.

The effective no-flux conditions at each interface mean that we can obtain the following global constraints

$$
\int_{r_{c 0}}^{r_{f}(\tau)} r^{2} x_{l}(r, \tau) \mathrm{d} r=\frac{1-r_{c 0}^{3}}{3}, \quad \int_{r_{c 0}}^{r_{f}(\tau)} r^{2} y_{l}(r, \tau) \mathrm{d} r=Y_{0} \frac{1-r_{c 0}^{3}}{3},
$$

valid over this timescale. If $x_{l}$ and $y_{l}$ become spatially uniform, which is typically the case for large $\tau$, these expressions determine the limiting behaviour

$$
r_{f} \sim\left(-\frac{\alpha}{T_{p}}+r_{c 0}^{3}\left(1+\frac{\alpha}{T_{p}}\right)\right)^{1 / 3}, \quad y_{l} \sim-\frac{Y_{0} T_{p}}{\alpha} \quad \text { as } \tau \rightarrow \infty .
$$

We use these results as the large- $\tau$ matching conditions for the next timescale. 
3.4. Osmotic timescale: $t=O(1 / \kappa)$. On the final timescale, the heat conduction and solute diffusion are very quick, so the temperature and concentration are spatially independent. This yields a significant reduction in the complexity of the system. The important processes are solute permeation through the cell membrane and the movement of this membrane. The freezing front can also advance into the liquid, and this is governed by two main effects. Firstly, as before, the CPA in the liquid medium is concentrated by the advancing freezing front shrinking the domain, this lowers the freezing point and slows the front movement. Secondly, as CPA permeates into the cell, the freezing front must advance more quickly to reduce the size of the liquid domain to conserve total CPA. This is because the liquid CPA concentration is at a constant level fixed by the freezing point of the liquid.

To formalize the above, we introduce the slow timescale $\zeta=\kappa t=O(1)$, from which we see that we can have $\mathrm{d} r_{c} / \mathrm{d} \zeta=O(1)$ and $\mathrm{d} r_{f} / \mathrm{d} \zeta=O(1)$. From (9), the thermal conduction is very quick and the temperature over this timescale is prescribed by the external boundary, resulting in $T_{s}=T_{l}=T_{p}(\zeta)$. From (10), solute diffusion is also very quick, leading to chemical concentration being independent of the spatial coordinate. Hence the freezing temperature at the freezing front (13a) tells us that within the liquid medium

$$
x_{l}=-\frac{T_{p}(\zeta)}{\alpha} .
$$

To determine the remaining time-dependent concentrations, we integrate each concentration equation over its respective domain, with the knowledge that each variable is independent of space at leading order.

This procedure results in the following governing equations

$$
\begin{array}{r}
\frac{\mathrm{d}}{\mathrm{d} \zeta}\left(r_{c}^{3} y_{c}\right)=0, \\
\frac{\mathrm{d}}{\mathrm{d} \zeta}\left(\left(r_{f}^{3}-r_{c}^{3}\right) y_{l}\right)=0, \\
\frac{\mathrm{d}}{\mathrm{d} \zeta}\left(r_{c}^{3} x_{c}\right)=-\frac{3 \omega r_{c}^{2}}{\kappa}\left(\frac{T_{p}(\zeta)}{\alpha}+x_{c}\right), \\
\frac{\mathrm{d}}{\mathrm{d} \zeta}\left(\left(r_{f}^{3}-r_{c}^{3}\right) \frac{T_{p}(\zeta)}{\alpha}\right)=-\frac{3 \omega r_{c}^{2}}{\kappa}\left(\frac{T_{p}(\zeta)}{\alpha}+x_{c}\right), \\
\frac{\mathrm{d} r_{c}}{\mathrm{~d} \zeta}=\sigma\left(\frac{T_{p}(\zeta)}{\alpha}+x_{c}\right)+y_{c}-y_{l},
\end{array}
$$

for the five remaining variables, $x_{c}, y_{c}, y_{l}, r_{c}$, and $r_{f}$, which are all solely functions of $\zeta$. The matching conditions for this system into the earlier timescale as $\zeta \rightarrow 0$ are as follows:

$$
\begin{array}{r}
x_{c}(0)=1, \quad y_{c}(0)=Y_{0}, \quad y_{l}(0)=\lim _{\zeta \rightarrow 0}\left(-\frac{Y_{0} T_{p}(\zeta)}{\alpha}\right), \\
r_{c}(0)=r_{c 0}, \quad r_{f}(0)=\lim _{\zeta \rightarrow 0}\left(-\frac{\alpha}{T_{p}(\zeta)}+r_{c 0}^{3}\left(1+\frac{\alpha}{T_{p}(\zeta)}\right)\right)^{1 / 3} .
\end{array}
$$

We are able to reduce this system from five ordinary differential equations to just two, for $x_{c}$ and $r_{c}$, as we now describe. The equations (31c) and (31d) can be combined to eliminate the right-hand sides and integrated directly, yielding an expression for global CPA conservation

$$
r_{c}^{3} x_{c}-\left(r_{f}^{3}-r_{c}^{3}\right) \frac{T_{p}}{\alpha}=1
$$

Rearranging (33) to obtain an expression for the position of the freezing front in terms of $x_{c}, r_{c}$, and $T_{p}$, and integrating the equations (31a) and (31b) directly, we obtain the algebraic relationships

$$
y_{c}=\frac{r_{c 0}^{3}}{r_{c}^{3}} Y_{0}, \quad y_{l}=\frac{1-r_{c 0}^{3}}{r_{f}^{3}-r_{c}^{3}} Y_{0}, \quad r_{f}=\left(r_{c}^{3}-\frac{\alpha}{T_{p}}\left(1-r_{c}^{3} x_{c}\right)\right)^{1 / 3} .
$$

Therefore, we can reduce the system (31) to the following two ODEs for $x_{c}$ and $r_{c}$

$$
\frac{\mathrm{d}}{\mathrm{d} \zeta}\left(r_{c}^{3} x_{c}\right)=-\frac{3 \omega r_{c}^{2}}{\kappa}\left(\frac{T_{p}}{\alpha}+x_{c}\right)
$$




$$
\frac{\mathrm{d} r_{c}}{\mathrm{~d} \zeta}=\sigma\left(\frac{T_{p}}{\alpha}+x_{c}\right)+Y_{0}\left(\frac{r_{c 0}^{3}}{r_{c}^{3}}+\frac{T_{p}}{\alpha} \frac{1-r_{c 0}^{3}}{1-r_{c}^{3} x_{c}}\right)
$$

with $x_{c}(0)=1$ and $r_{c}(0)=r_{c 0}$. The system (35) is similar to other ODE models that have been used in the past to model cryopreservation (e.g. $[6,9,13])$, based on the Kedem-Katchalsky (KK) framework for the volume and intracellular solute concentration of cells during cryopreservation [26, 29]. Our asymptotic analysis shows that (35) is the relevant KK model for the set-up we consider over longer timescales once the temperature and concentration have become uniform in each domain. The differences between our model and the standard KK model are: (1) we consider an additional solute - the ion concentration, and (2) our model has systematically accounted for the CPA and ion concentrations both internally and externally to the cell, as well as their coupling to the prescribed temperature at the external boundary.

Noting that $T_{p} \rightarrow-1$ when the final freezing temperature is reached, the final equilibrium values are given by

$$
x_{c}, x_{l}=\frac{1}{\alpha}, \quad y_{c}, y_{l}=\frac{Y_{0}}{\alpha}, \quad r_{c}=r_{c 0} \alpha^{1 / 3}, \quad r_{f}=\alpha^{1 / 3}
$$

as verified in Figures 2-4.

3.4.1. Small $r_{c 0}$. We note that, in practice, the value of $r_{c 0}$ is fairly small, and hence $r_{c 0}^{3}$ and $r_{c}^{3}$ can be ignored relative to larger terms in (34)-(35). Making that simplification, we find

$$
y_{l}=-\frac{T_{p} Y_{0}}{\alpha}, \quad r_{f}=\left(-\frac{\alpha}{T_{p}}\right)^{1 / 3},
$$

and (35b) can be approximated by

$$
\frac{\mathrm{d} r_{c}}{\mathrm{~d} \zeta}=\sigma\left(\frac{T_{p}}{\alpha}+x_{c}\right)+Y_{0}\left(\frac{r_{c 0}^{3}}{r_{c}^{3}}+\frac{T_{p}}{\alpha}\right)
$$

4. Distinguished limits for the cooling rate. Now that we have described the reduced models that hold on each timescale of the problem, we now consider how the behaviour depends on the cooling rate, $\beta$. The dimensionless cooling timescale is $1 / \beta$, and we consider three distinguished limits for $\beta$, where $\beta=O(1)$, $O\left(D_{l}^{x}\right)$, and $O(\kappa)$ in turn, proceeding from fastest to slowest cooling rate. We also consider the sub-limits of $\beta \gg 1$ and $\beta \ll \kappa$ in their respective relevant distinguished limits.

4.1. Rapid cooling: $\beta=O(1)$. In the case of rapid cooling, where $\beta=O(1)$, the temperature evolves on the conductive timescale $t=O(1)$ and is outlined in $\S 3.2$. Analytic expressions for the temperature over this timescale are given in (22) and (71). The temperature tends to its uniform final value $T=-1$ by the end of this timescale.

Later, when $t=O\left(1 / D_{l}^{x}\right)$, the problem is governed by (24) - (27), with the general condition (26a) given the specific form

$$
x_{l}=\frac{1}{\alpha} \quad \text { for } r=r_{f}(\tau) .
$$

This problem determines the diffusive evolution of the solute concentration and the freezing front evolution. From the integral constraints (29), we have the following large-time behaviour of the reduced moving boundary problem:

$$
x_{l} \rightarrow \frac{1}{\alpha}, \quad y_{l} \rightarrow \frac{Y_{0}}{\alpha}, \quad r_{f} \rightarrow\left(\alpha+r_{c 0}^{3}(1-\alpha)\right)^{1 / 3} \quad \text { as } \tau \rightarrow \infty .
$$

Finally, on the osmotic timescale, outlined in $\S 3.4,(30)$ and (34) give

$$
x_{l}=\frac{1}{\alpha}, \quad y_{c}=\frac{r_{c 0}^{3}}{r_{c}^{3}} Y_{0}, \quad y_{l}=\frac{1-r_{c 0}^{3}}{\alpha-r_{c}^{3} x_{c}} Y_{0}, \quad r_{f}=\left(\alpha+r_{c}^{3}\left(1-x_{c}\right)\right)^{1 / 3} .
$$


Recalling the definition $\zeta=\kappa t$, the governing ODEs (35) for $x_{c}$ and $r_{c}$ can be simplified to

$$
\begin{array}{r}
\frac{\mathrm{d}}{\mathrm{d} \zeta}\left(r_{c}^{3} x_{c}\right)=\frac{3 \omega r_{c}^{2}}{\alpha \kappa}\left(1-\alpha x_{c}\right), \\
\alpha \frac{\mathrm{d} r_{c}}{\mathrm{~d} \zeta}=-\sigma\left(1-\alpha x_{c}\right)+Y_{0}\left(\frac{\alpha r_{c 0}^{3}}{r_{c}^{3}}-\frac{1-r_{c 0}^{3}}{1-r_{c}^{3} x_{c}}\right),
\end{array}
$$

with $x_{c}(0)=1$ and $r_{c}(0)=r_{c 0}$. Thus, the large-time dynamics for rapid cooling all collapse onto the solutions of (42), as predicted by the collapse of the faster cooling rates onto a single curve in Figures $5 \mathrm{a}$ and 6 a.

4.1.1. Very rapid cooling: $\beta \gg 1$. It is informative to note the simplifications that occur when the cooling timescale is much faster than the heat conduction timescale. In this asymptotic limit, the reductions occur over the first timescale where $t=O(1)$, and $T_{p}^{\prime}(t) \approx-(1-\alpha) \delta(t)$, where $\delta(t)$ is the Dirac delta function. Hence $T_{l}$, given in (22), becomes

$$
T_{l}=-1-\frac{2(1-\alpha)}{r} \sum_{n=1}^{\infty} \frac{(-1)^{n} \exp \left(-k_{l} n^{2} \pi^{2} t\right)}{n \pi} \sin n \pi r
$$

Solutions for the chemical concentration in the boundary layer in this limit are given in Appendix D.1.

4.2. Moderate cooling: $\beta=O\left(D_{l}^{x}\right)$. In the case of moderate cooling where $\beta=O\left(D_{l}^{x}\right)$, the thermal conductivity timescale $t=O(1)$ (outlined in $\S 3.2$ ) is now fairly uninteresting, since the prescribed temperature change is small on this timescale. To leading order, nothing happens until the $t=O\left(1 / D_{l}^{x}\right)$ timescale on which both temperature and solute evolve.

Over this timescale, the temperature is spatially uniform, with $T_{l}=T_{s}=T_{p}(\tau)$, and it decreases from its initial value of $-\alpha$ to its final value of -1 . The reduced problem, given in (24)-(27), consists of a partial differential equation for $x_{l}$ with one moving boundary for $r_{f}$. Since $T_{p}(\tau)$ reaches the final value of -1 , the large-time state attained over this timescale is the same as for the rapid cooling case in $\S 4.1$, given in (40). From this point onwards the dynamics of the moderate cooling case are exactly the same as those in $\S 4.1$, as described by (41)-(42).

4.3. Slow cooling: $\beta=O(\kappa)$. In the case of slow cooling where $\beta=O(\kappa)$, both the thermal conductivity and solute diffusion timescales (outlined in $\$ 3.2$ and $\$ 3.3$ ) are uninteresting. Over both of these timescales, the leading-order system is essentially solved by the initial conditions (17). The interesting timescale for this case is where $\zeta=\kappa t=O(1)$, described in $\S 3.4$. Over this timescale, the system is governed by (30), (34)-(35).

4.3.1. Very slow cooling: $\beta \ll \kappa$. Finally, it is also of interest to determine the further reduced system when the cooling rate is even smaller than in the distinguished limit of slow cooling, that is when $\beta \ll \kappa$. In this case the dynamics become quasi-steady. The solution is given parametrically in terms of $T_{p}$

$$
x_{c}=x_{l}=-\frac{T_{p}}{\alpha}, \quad y_{c}=y_{l}=-\frac{T_{p} Y_{0}}{\alpha}, \quad r_{c}=r_{c 0}\left(-\frac{\alpha}{T_{p}}\right)^{1 / 3}, \quad r_{f}=\left(-\frac{\alpha}{T_{p}}\right)^{1 / 3} .
$$

The latter two solutions for the membrane and freezing front position are shown in Figures 5b and $6 \mathrm{~b}$ by the dashed lines.

4.4. Overview of asymptotic results. Our asymptotic solutions consist of the analytic results for the liquid temperature (22) and ice temperature $T_{s}=T_{p}$, the reduced partial differential equation for CPA concentration within the liquid medium with a moving boundary at the freezing front (24)-(27) over the intermediate timescale, and the heavily reduced system of a coupled set of ordinary differential equations for the chemical concentrations and moving boundaries (35) over the slow timescale.

To summarize, the ice temperature is always spatially independent and equal to the external temperature for all cooling rates. The liquid temperature can be spatially dependent for fast cooling rates over the heat conduction timescale (22), but is spatially independent in all other cases. The chemical concentrations and motions of the moving boundaries are strongly coupled to one another. While these quantities are forced by 

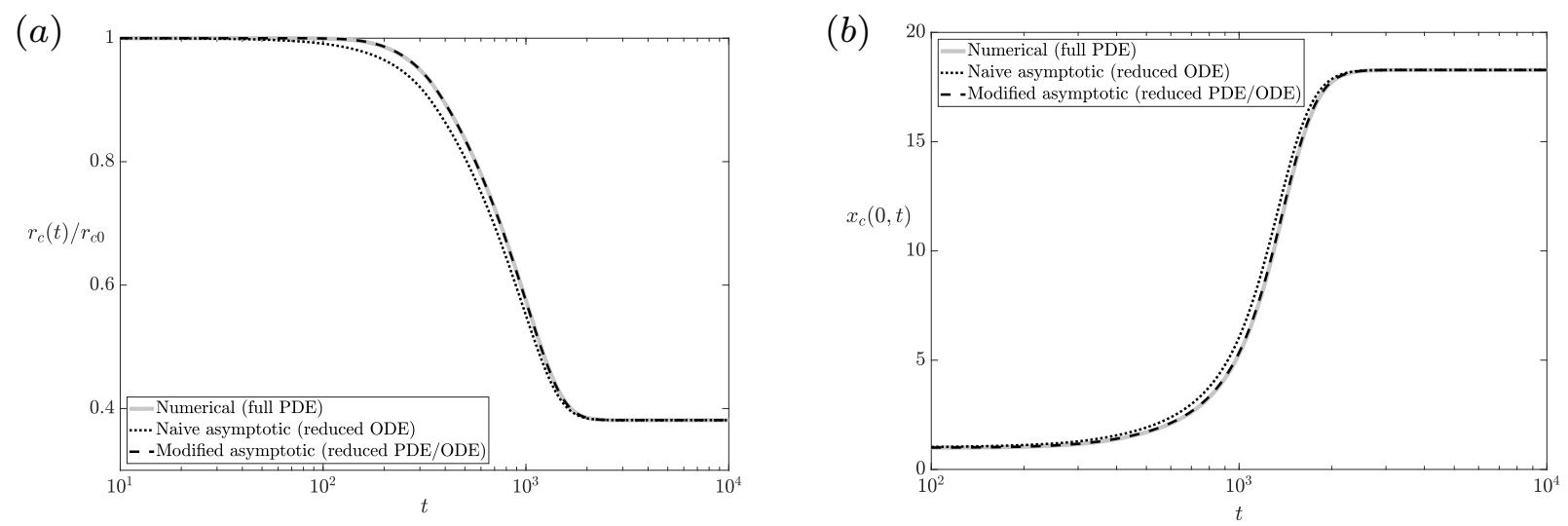

FiG. 8. A comparison of our numerical and asymptotic solutions for $(a) r_{c}$ and $(b) x_{c}(0, t)$. We use a dimensionless cooling rate of $\beta=1$, and the remaining parameters from Table 3. The naive asymptotic solution is from (30), (34)-(35), the modified asymptotic solution is from (45). The naive asymptotic solutions agree well with the numerical solution, whereas the modified asymptotic solutions are almost indistinguishable.

the decrease in temperature, the movement of the freezing front dictates the chemical balance. The Stefan condition (13d) is more correctly interpreted for this problem as a statement about the small flux of heat from the freezing front to the ice phase, rather than a statement about the balance of heat fluxes with the front velocity. The cell membrane movement and the CPA concentration within the cell only vary from their initial values over the slow timescale, no matter what cooling rate is imposed. This means that faster cooling rates will result in significant differences in CPA concentration within the cell compared to at the freezing front. This will cause a difference in the freezing temperature at each location, and will therefore result in supercooling within the cell. This is discussed in more detail in $\S 5$.

These asymptotic solutions provide fast and accurate approximations of the numerical solutions when compared with the size of the cell and the CPA concentration within the cell, as shown in Figure 8. These variables are the key quantities for determining cell damage, and we will exploit our asymptotic solutions in $\S 5$ to quantify cell damage in a computationally efficient manner. Such insights allow us to numerically implement boundary conditions in a manner that agrees with the flow of information in the system. There is a slight discrepancy between the asymptotic and numerical results for intermediate times in Figure 8, we discuss how to resolve this in the next section.

4.5. Modified asymptotic solutions. We note that there is a discrepancy between the osmotic timescale for faster cooling predicted by the asymptotic analysis $\left(t=O(1 / \kappa)=O\left(2 \times 10^{5}\right)\right)$, and that seen in Figures 3-4, where the cell membrane movement occurs over $t=O\left(10^{3}\right)$. In terms of the physics of the problem, this occurs because the extracellular solute concentration is not uniform during the early part of the osmotic timescale. However, we shall demonstrate in this section that a careful incorporation of the required spatial dependence does provide the required correction.

In terms of the asymptotic methodology we use, this discrepancy occurs because, as is often the case with asymptotic methods, there are quantities we treat as $O(1)$ that combine to become large in practice. For this problem, we can obtain a modified osmotic timescale directly from (12c). As the CPA concentration difference across the membrane can be up to $1 / \alpha$ and the cell size is $O\left(r_{c 0}\right)$, a more accurate osmotic timescale is $t=O\left(r_{c 0} \alpha / \kappa\right)=O\left(10^{3}\right)$, which does agree with Figures 3-4. While the osmotic timescale should therefore start to merge with the solute diffusion timescale $\left(t=O\left(1 / D_{l}^{x}\right)=O\left(10^{3}\right)\right)$, this does not occur completely since the diffusion timescale is itself shortened by the reduction in the liquid domain size, largely keeping these timescales separate. The practical effect of this is that there is a slight merging of these two timescales, and this causes the small difference between the numerical and asymptotic solutions in Figure 8. In essence, the cell motion is slightly slower than predicted by the asymptotic analysis. This is because the CPA concentration is actually lower at the cell membrane than at the freezing front early in the osmotic timescale. This results in smaller forcing of the cell motion than predicted by the asymptotic analysis which assumes a sharp separation of timescales leading to the CPA concentration being spatially 
uniform throughout the osmotic timescale. We refer to the original set of asymptotic solutions as the 'naive' asymptotic results.

To fix the discrepancy, we can formulate an appropriate reduced composite equation using the fact that $x_{c}$ is approximately independent of space for all time. To do this, we first solve for $x_{l}(r, \tau)$ over the medium time, using the reduced PDE system derived in $\S 3.3$. This allows us to determine $x_{l}\left(r_{c 0}, \tau\right)$ at the cell membrane (noting that the cell membrane is stationary over this timescale), and we define this quantity as $f(\zeta):=x_{l}\left(r_{c 0},\left(D_{l}^{x} / \kappa\right) \zeta\right)$. Then, we may derive the reduced ODE system

$$
\begin{array}{r}
\frac{\mathrm{d}}{\mathrm{d} \zeta}\left(r_{c}^{3} x_{c}\right)=-\frac{3 \omega r_{c}^{2}}{\kappa}\left(x_{c}-f(\zeta)\right), \\
\frac{\mathrm{d} r_{c}}{\mathrm{~d} \zeta}=\sigma\left(x_{c}-f(\zeta)\right)+Y_{0}\left(\frac{r_{c 0}^{3}}{r_{c}^{3}}-f(\zeta) \frac{1-r_{c 0}^{3}}{1-r_{c}^{3} x_{c}}\right),
\end{array}
$$

with $x_{c}(0)=1$ and $r_{c}(0)=r_{c 0}$, for the osmotic timescale. This is a direct modification of (35), replacing $-T_{p}(\zeta) / \alpha$ with $f(\zeta)$, the pre-computed function described above. We refer to these as the 'modified' asymptotic results. The system (45) can also be thought of as a modified Kedem-Katchalsky (KK) ODE model [26, 29], valid over the medium and long timescales, requiring the solution of the reduced PDE system derived in $\S 3.3$ as an input.

Using (45), our modified asymptotic results show excellent agreement with the full numerical results (Figure 8), allowing us to be confident in both our numerical and asymptotic solutions. While this modified asymptotic solution is slightly more computationally intensive than simply obtaining the solution of the ODE (35), it remains around 500 times faster than a full numerical solution.

5. Cell damage. In this section, we use our results to estimate the potential damage caused to cells during freezing. The two main mechanisms of damage are due to intracellular ice formation, and chemical toxicity. The former is triggered by supercooling, a quantity we are able to calculate directly from our model (Figure 9a). We observe that cells can experience significant levels of supercooling. Our first metric quantifies intracellular ice formation by integrating the total supercooling over time. As cytoplasm can tolerate a certain level of supercooling before freezing occurs, we build this into our metric by only counting

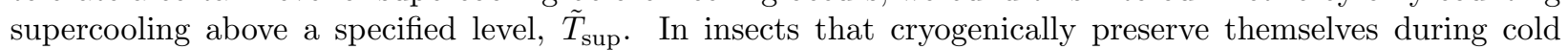
weather, $\tilde{T}_{\text {sup }} \approx 20^{\circ} \mathrm{C}[50]$, and so we use this value here for definiteness, but we emphasize that this value can be varied if required.

With the assumptions outlined above, our metric for cell damage due to supercooling is

$$
\mathcal{S}:=\int_{0}^{\infty} \frac{4}{3} \pi r_{c}^{3}\left[T_{f}\left(x_{c}(0, t)\right)-T_{l}(0, t)-T_{\text {sup }}\right]_{+} \mathrm{d} t,
$$

where $[f(x)]_{+}$denotes the positive part of $f(x)$ and $T_{\text {sup }}=\tilde{T}_{\text {sup }} /\left(\tilde{T}_{f 0}-\tilde{T}_{\text {end }}\right) \approx 0.29$ is the dimensionless level of safe supercooling within cytoplasm. We have integrated over the cell volume approximating the supercooling by its value at the cell centre, since our solutions show that the supercooling is essentially independent of space within the cell. As one might expect, the damage due to supercooling increases as the cooling rate increases (Figure 10a).

To quantify cell damage due to chemical toxicity, we assume that toxicity accumulates over time at a rate proportional to CPA concentration and cell volume, but we note that a more general toxicity function could incorporate effects due to the increased concentration of the impermeable chemical species as well. We see that lower cooling rates lead to higher CPA concentrations at the cell centre for the same external temperatures (Figure 9b), which suggests that toxicity is likely to be more of a concern for slow cooling, agreeing with experimental observation. We assume that the rate of proportionality for toxicity satisfies an Arrhenius-type temperature dependence with activation energy $\tilde{E}_{a}$ [14]. To this end, we use the following metric to quantify CPA toxicity

$$
\mathcal{T}:=\int_{0}^{\infty} \frac{4}{3} \pi r_{c}^{3} x_{c}(0, t) e^{-E_{a} /\left(1+\nu T_{l}(0, t)\right)}\left[T_{l}(0, t)-T_{\text {met }}\right]_{+} \mathrm{d} t,
$$

where $E_{a}=\tilde{E}_{a} /\left(\tilde{R} \tilde{T}_{f 0}\right)$ is a dimensionless activation energy (we use $E_{a}=40$, corresponding to $\tilde{E}_{a}=90.6 \mathrm{~kJ}$ $\left.\mathrm{mol}^{-1}\right)$, and $\nu=\left(\tilde{T}_{f 0}-\tilde{T}_{\text {end }}\right) / \tilde{T}_{f 0}$. While the introduction of this Arrhenius factor means that CPA toxicity 

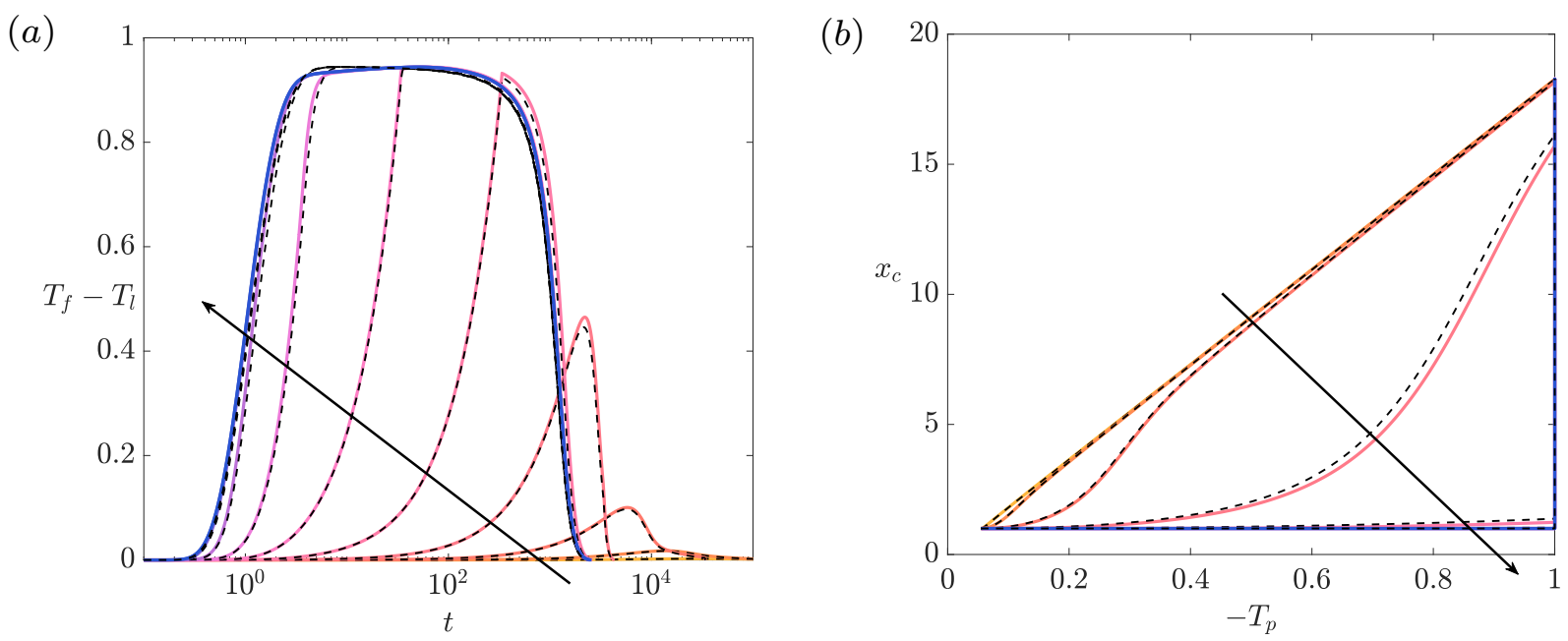

Fig. 9. Proxies for cell damage at the cell centre. (a) Supercooling $T_{f}\left(x_{c}(0, t)\right)-T_{l}(0, t)$ versus time. (b) CPA concentration $x_{c}(0, t)$ versus external temperature. The different lines correspond to different values of the cooling rate; we use $\beta=2.8 \times 10^{k}$, where $k=\{-7, \ldots, 3\}$ (corresponding to dimensional cooling rates ranging between $10^{-4}-10^{6} \mathrm{~K} \mathrm{~s}^{-1}$ ). The solid lines are numerical solutions and the black dashed lines are asymptotic solutions, using the analytic result for the temperature (22) from the fast timescale and the naive asymptotic solution (30), (34)-(35) from the slow timescale. The arrows denote increasing cooling rate.
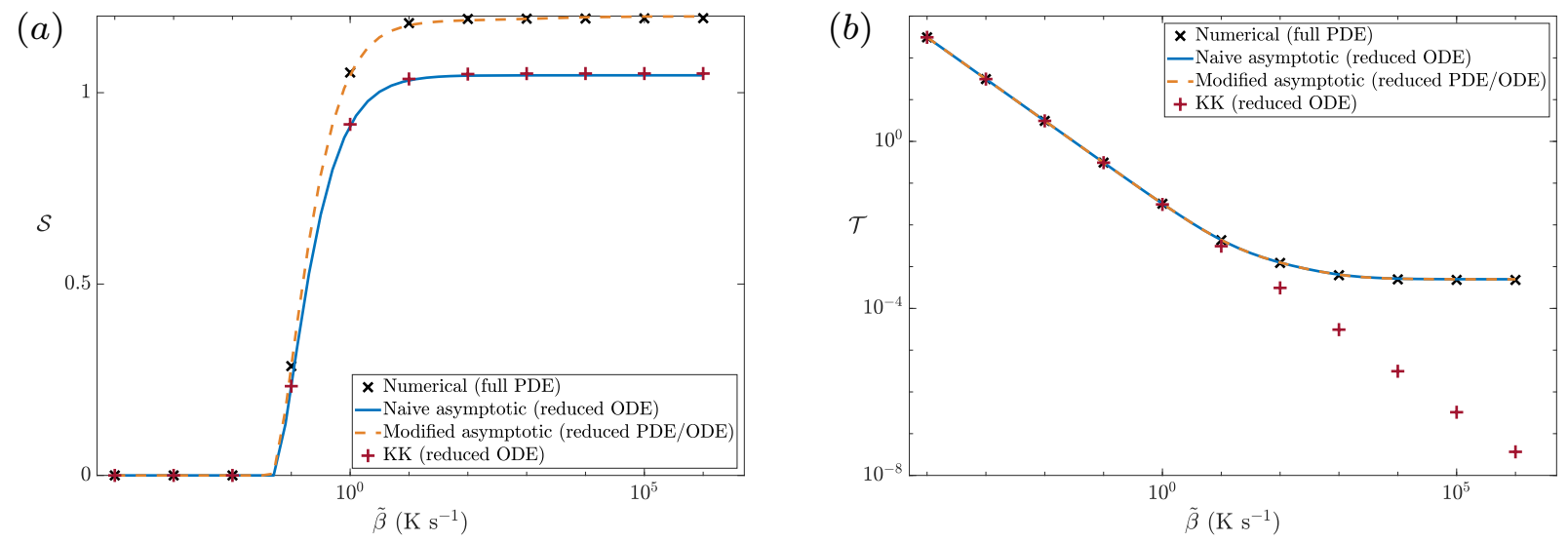

FIG. 10. The metrics for cell damage in our system, (a) $\mathcal{S}$ and (b) $\mathcal{T}$, using $T_{\text {sup }}=0.29, E_{a}=40$, and $T_{m e t}=-0.9$.

decreases as the temperature decreases, it is also helpful to impose that the CPA toxicity explicitly falls to zero when the temperature falls below a certain level, defined in dimensionless terms as $T_{\text {met }}$. This ensures that we do not accrue infinite toxicity as $t \rightarrow \infty$. We impose a value of $T_{\mathrm{met}}=-0.9$ here for definiteness, but again emphasize that this can be varied if required. We note that the metric $\mathcal{T}$ is similar to the temperature-dependent toxicity cost function used in Davidson et al. [14] to optimise operating conditions during cryopreservation, itself an adaptation of the toxicity cost function from [6, 7, 13], for example. However, we consider the integrand to be linearly proportional to CPA concentration rather than as a noninteger power, and we scale toxicity with the cell volume. We note that the damage due to CPA toxicity increases as the cooling rate decreases (Figure 10b), in agreement with physical intuition and experimental observation.

When we compare the damage predicted by our asymptotic results to that predicted by our numerical solutions, we note that while there is excellent agreement for $\mathcal{T}$, the asymptotic results for $\mathcal{S}$ systematically predict a lower damage from supercooling if we use the naive asymptotic results discussed in $\S 4.4$ (Figure 


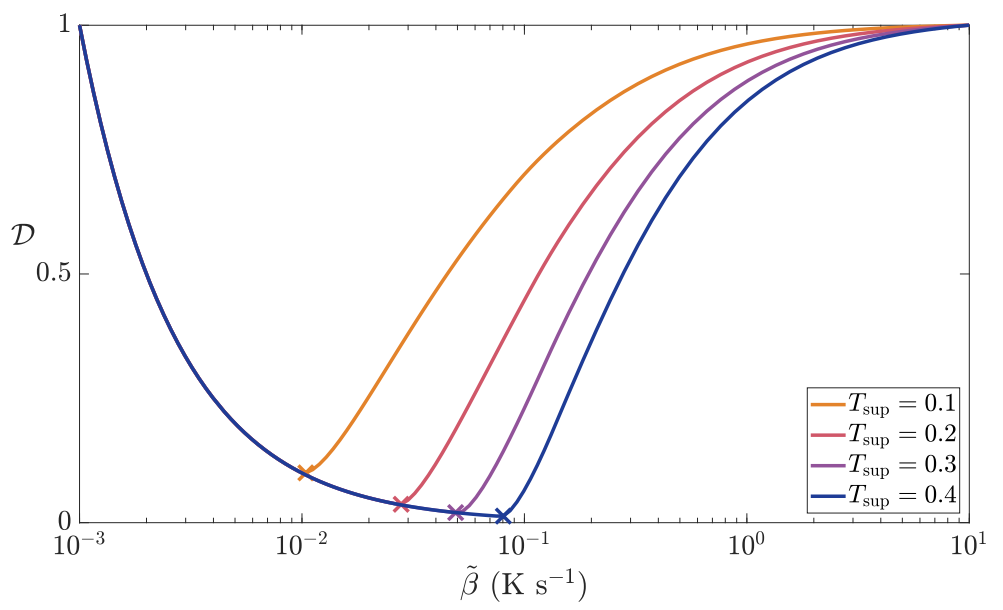

FIG. 11. The cell damage function $\mathcal{D}$, defined in (47) with $\lambda$ and $\mu$ chosen to normalize the maximum values of $\mathcal{S}$ and $\mathcal{T}$ as described in the text, versus cooling rate $\beta$. We show results for different cytosol tolerances to supercooling ( $\left.T_{\text {sup }}\right)$, which lead to different optimal cooling rates which minimize $\mathcal{D}$, denoted by a cross.

10). However, the modified asymptotic results show excellent agreement for both. In Figure 10, we also plot the damage predicted by using the model (35) (for the slow timescale) for all time. We refer to this as the Kedem-Katchalsky (KK) model. As one might expect, the results from this are very similar to those of the naive asymptotic results, which differ from the KK model by using the analytic solution for the temperature (22) over the fast timescale, in addition to (35) over the slow timescale. This is because even though there are significant differences between the KK and naive asymptotic results in the liquid and ice regions, the only difference between our naive asymptotic results and the KK equations at the cell centre is the temperature over the fast timescale for fast cooling rates. This explains the discrepancy in $\mathcal{T}$ for faster cooling rates; the KK equations over-estimate the rate of heat propagation to the cell centre at faster cooling rates.

Hence, we are able to efficiently compute $\mathcal{S}$ and $\mathcal{T}$ as metrics for cell damage in the system. For given cell membrane permeability parameters and appropriate weightings of each metric, we can use these results to compute optimal cooling rates, using a combined damage function,

$$
\mathcal{D}=\lambda \mathcal{S}+\mu \mathcal{T} .
$$

For example, if we sweep between $\tilde{\beta} \in\left[10^{-3}, 10^{1}\right] \mathrm{K} \mathrm{s}^{-1}$ and choose $\lambda$ and $\mu$ to normalize $\mathcal{S}$ and $\mathcal{T}$ such that their highest values are equal to one, we see that our damage function predicts a minimum in $\mathcal{D}$ at an intermediate cooling rate (Figure 11). The optimal cooling rate increases as the tolerance to supercooling increases. The framework we have developed in this paper allows us to quantify the optimal cooling rate for given cell parameters.

6. Discussion. We have derived and solved a mathematical model for the cryopreservation of a cell immersed in a liquid medium, using a combination of numerical and asymptotic methods. Our model accounts for spatial variation of temperature and chemical concentrations, and for the motion of a freezing front and cell membrane. The system is fully coupled, since the presence of cryoprotective agent (CPA) lowers the freezing point, and the membrane movement is generated by an osmotic force of chemical concentration difference across the membrane. Investigating this system has provided insight into how the coupled physical mechanisms underlying cryopreservation combine during the freezing process, and when they cause cell damage. To this end, we have introduced two different damage metrics to infer the implicit cell damage caused by freezing. The first quantifies the cumulative supercooling occurring within the cell as a proxy for intracellular ice formation. The second measures the cumulative CPA toxicity occurring within the cell. We note that it is impossible to globally optimize both of these metrics separately since it is observed that faster cooling has a greater chance of intracellular ice formation and slower cooling suffers from greater CPA toxicity. Our metrics allow us to quantify these observations, and show that there is an 'optimal' cooling rate, which will depend on the cell properties and the operating conditions of the cryopreservation process. These results are consistent with the well-known 'two-factor hypothesis' of freezing injury [39]. Our work 
provides a computationally efficient framework from which to determine this optimal cooling rate.

It would be straightforward to modify both the toxicity and supercooling metrics to account for different cell biology or sensitivity to the ion concentration, for example. For simplicity, we have not explicitly considered the mechanism of intracellular ice formation; we only allow ice to form from a nucleation surface at the external boundary. Explicitly accounting for the mechanism of supercooling and new crystal nucleation with an unstable freezing front could lead to mushy layers near the front, consisting of ice and water. This would be an interesting extension of the model we present in this paper.

We have focused on the freezing process of cryopreservation here, but note that there are also interesting physical problems arising in the thawing stage. As our model is inherently nonlinear, a reversal of the freezing process will not result in a strict reversal of the dynamics, and would be an interesting problem in its own right.

Moreover, we note that while this work is for a single cell, a significant application of cryopreservation is for biological tissue. Safely freezing larger tissues remains a current challenge in the field. Our model could be extended to a tissue comprising many cells using the mathematical technique of homogenization [10, 22]. However, care must be taken in this upscaling procedure due to the moving boundaries in the problem - one could follow the methodology of $[12,44]$, for example.

The higher cooling rates we consider in this paper start to touch on the realm of vitrification, where a liquid is cooled rapidly past its glass transition point so as to form a non-crystalline amorphous solid rather than a crystalline ice [43]. While vitrification is not a focus of this work, and we do not account for the mechanisms of vitrification in the model, we note that a drop in CPA diffusivity associated with lowering temperature can be accounted for using the results in Appendix A. In this Appendix, we provide systematically reduced systems for the cryopreservation of a single cell where the cell parameters are temperature dependent. Although the solutions will vary due to this temperature dependence, the asymptotic structure of the problem remains the same.

Finally, we note that this work has the potential to guide cryopreservation protocols for the freezing of single cells. Our methodology allows us to account for the spatial variations inherent to the system at faster cooling rates, and to systematically reduce the system over the different timescales. This approach allows us to derive asymptotic solutions which largely circumvent the issue of expensive parameter sweeps, resulting in a computationally efficient framework to compute the cell damage for given cell properties and operating conditions.

Data deposition. The computational code we developed to solve this model (outlined in Appendix B) is openly available at https://github.com/m-dalwadi/Mathematical-model-cryopreservation.

\section{Appendix A. Temperature-dependent coefficients.}

In this Appendix we outline how the problem changes when we allow the following chemical transport parameters to depend on temperature: $D_{c}^{x}, D_{c}^{y}, D_{l}^{x}, D_{l}^{y}, \omega, \kappa$. Though we carry out this analysis for general temperature-dependent coefficients, it may be helpful to think of the membrane coefficients having an Arrhenius-type dependence on the temperature. For the diffusivities, one may consider a modified Arrheniustype dependence, using the Stokes-Einstein equation for diffusivity, and an Arrhenius-type dependence for the viscosity.

In general, we expect all these parameters to decrease as the temperature decreases. Looking at activation energies [8, 15], we note that the activation energies for diffusivities are smaller than those for membrane permeability. Hence, the distinct timescales in $\S 3$ will separate rather than coalesce as the temperature decreases, maintaining the asymptotic structure that we identified in the main text. Hence, we are able to present the asymptotic solution to the generalized problem over the three important timescales identified in the main text as a simple extension of $\S 3$.

A.1. Thermal conduction timescale: $t=O(1)$. Over this timescale, the solution largely proceeds as in §3.2. For precision, we use the diffusivity of CPA in water at the initial temperature $D_{0}:=D_{l}^{x}(-\alpha)$ as our small (constant) parameter, and this replaces the $D_{l}^{x}$ in $\S 3.2$. In this case, the system is governed by the same temperature solutions (22) and (71). The chemical transport equations will be the early-time versions of those given in the next subsection.

A.2. Solute diffusion timescale: $t=O\left(1 / D_{0}\right)$. Over this timescale, the solution largely proceeds as in $§ 3.3$. We will again use the diffusivity of CPA in water at the initial temperature $D_{l}^{x}(-\alpha)$ as our small 
(constant) parameter, replacing the $D_{l}^{x}$ in $\S 3.3$. This means that we use $\tau=D_{0} t=O(1)$ as our timescale. The temperature is given by $T_{s}=T_{l}=T_{p}(\tau)$, but now the chemical transport problem is governed by

$$
\frac{\partial x_{l}}{\partial \tau}=\nabla \cdot\left(\frac{D_{l}^{x}}{D_{0}} \nabla x_{l}\right), \quad \frac{\partial y_{l}}{\partial \tau}=\nabla \cdot\left(\frac{D_{l}^{y}}{D_{0}} \nabla y_{l}\right) \quad \text { for } r_{c 0}<r<r_{f}(\tau),
$$

instead of (24). At the cell membrane, we have the no-flux versions of (12a)-(12b), which are

$$
\frac{D_{l}^{x}}{D_{0}} \frac{\partial x_{l}}{\partial r}+x_{l} \frac{\mathrm{d} r_{c}}{\mathrm{~d} \tau}=0, \quad \frac{D_{l}^{y}}{D_{0}} \frac{\partial y_{l}}{\partial r}+y_{l} \frac{\mathrm{d} r_{c}}{\mathrm{~d} \tau}=0 \quad \text { for } r=r_{c}(\tau)
$$

At the freezing front, we have the Dirichlet condition for the CPA concentration (26a), and the following conditions

$$
\frac{D_{l}^{x}}{D_{0}} \frac{\partial x_{l}}{\partial r}+x_{l} \frac{\mathrm{d} r_{f}}{\mathrm{~d} \tau}=0, \quad \frac{D_{l}^{y}}{D_{0}} \frac{\partial y_{l}}{\partial r}+y_{l} \frac{\mathrm{d} r_{f}}{\mathrm{~d} \tau}=0 \quad \text { for } r=r_{f}(\tau)
$$

to replace (26b) and (26c). The matching conditions for this system into the earlier timescale as $\tau \rightarrow 0$ are the same as in $\S 3.3$, given by (27) away from the freezing front, and addressed in the next section when the matching conditions near the freezing front may become relevant.

A.3. Osmotic timescale: $t=O\left(1 / \kappa_{0}\right)$. Over this timescale, the solution largely proceeds as in $\S 3.4$. We now use the hydraulic conductivity of the cell membrane at the initial temperature $\kappa_{0}:=\kappa(-\alpha)$ as our small parameter, replacing the $\kappa$ in $\S 3.4$. This means that we use $\zeta=\kappa_{0} t=O(1)$ as our timescale.

The temperature of the system is given by $T_{s}=T_{l}=T_{p}(\zeta)$, and the CPA concentration in the water phase is given by (30). The procedure to determine a closed system for the remaining variables is similar to that in $\S 3.4$. This means that $x_{c}, y_{c}, y_{l}$, and $r_{f}$ are given by (30) and (34), while the remaining generalized system is given by

$$
\frac{\mathrm{d}}{\mathrm{d} \zeta}\left(r_{c}^{3} x_{c}\right)=-\frac{3 \omega r_{c}^{2}}{\kappa_{0}}\left(\frac{T_{p}(\zeta)}{\alpha}+x_{c}\right), \quad \frac{\mathrm{d} r_{c}}{\mathrm{~d} \zeta}=\frac{\kappa}{\kappa_{0}}\left[\sigma\left(\frac{T_{p}(\zeta)}{\alpha}+x_{c}\right)+Y_{0}\left(\frac{r_{c 0}^{3}}{r_{c}^{3}}+\frac{T_{p}}{\alpha} \frac{1-r_{c 0}^{3}}{1-r_{c}^{3} x_{c}}\right)\right] .
$$

for the variables, $x_{c}, y_{c}$, and $r_{c}$, which are all solely functions of $\zeta$. The 'initial' conditions for this system as $\zeta \rightarrow 0$ are $x_{c}(0)=1$ and $r_{c}(0)=r_{c 0}$. Finally, we note that in the case where cooling occurs over a longer timescale than membrane movement $\left(\beta \ll \kappa_{0}\right)$, the temperature dependence of the parameters becomes irrelevant for the system dynamics.

\section{Appendix B. Landau transformation to three fixed domains.}

In this section we make a transformation to turn the Laplacian in our governing equations from an operator acting on a spherical coordinate system to a Cartesian one, and we perform the Landau transformation [30] to map the moving-boundary problem into a fixed domain problem. Both of these transformations will facilitate a numerical solution.

To transform the Laplacian in our governing equations, we introduce the new dependent variables

$$
\left(T_{l}, T_{s}, x_{c}, y_{c}, x_{l}, y_{l}\right)=\frac{1}{r}\left(\Theta_{l}, \Theta_{s}, X_{c}, Y_{c}, X_{l}, Y_{l}\right)
$$

In addition, we note that it is helpful for the numerical simulation to split $\Theta_{l}$ into $\Theta_{l, c}$ and $\Theta_{l, l}$, which hold in the cell and liquid domain, respectively. At the cell membrane, we couple these two new variables by imposing continuity of temperature and heat flux. We note that if $f=F / r$, then $f^{\prime}=F^{\prime} / r-F / r^{2}$, and therefore our governing equations and boundary conditions must be adjusted accordingly. We outline them below, after one additional transformation.

To transform the moving-boundary problem into three fixed domains, we introduce new independent variables based on the formulation

$$
\xi=g\left(\frac{r-a(t)}{b(t)-a(t)}\right)
$$




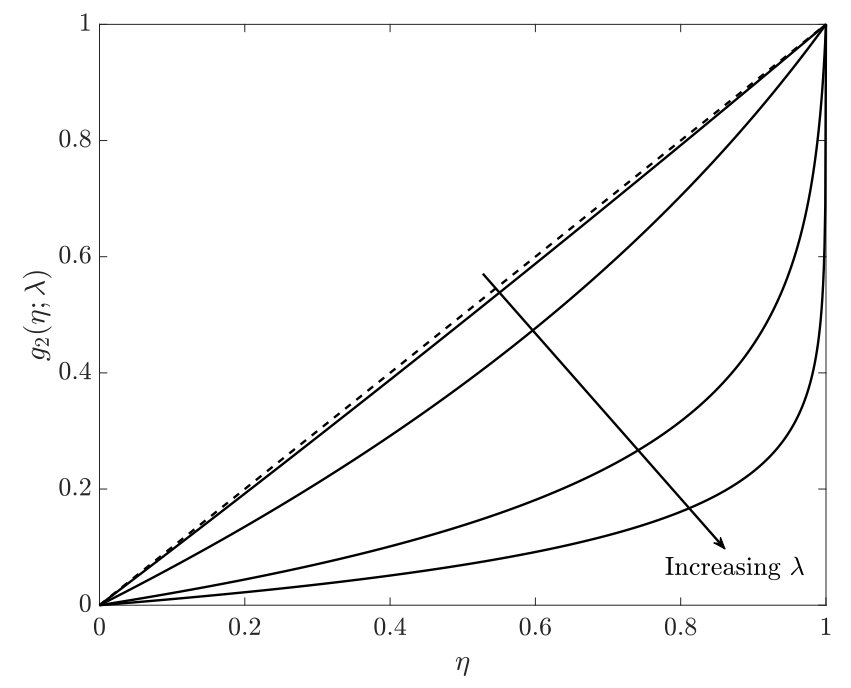

FIG. 12. The nonlinear monotonic function $g_{2}(\eta ; \lambda)$ (Table 4), used in the transformation of the domain $r_{c}(t)<r<r_{f}(t)$ to resolve the boundary layers near $r=r_{f}(t)$. The dotted line corresponds to $g_{2}\left(\eta ; 0^{+}\right)=\eta$, and the solid lines correspond to $\lambda=0.1,1,5,10$. Hence, we see that a uniform grid spacing of $g_{2}$ corresponds to more grid points near $\eta=1$, with this effect being amplified as $\lambda$ increases.

where $r \in(a(t), b(t))$, and $g$ is a monotonic increasing function, with $g(0)=0$ and $g(1)=1$. The purpose of $g$ is to allow a uniform discretization of $\xi$ to provide a non-uniform discretization of $r$, allowing for finer resolutions near boundary layers. For our problem, we use

$$
\begin{array}{r}
\xi_{1}=g_{1}\left(\frac{r}{r_{c}(t)}\right) \quad \text { for } 0<r<r_{c}(t), \\
\xi_{2}=g_{2}\left(\frac{r-r_{c}(t)}{r_{f}(t)-r_{c}(t)} ; \lambda\right) \quad \text { for } r_{c}(t)<r<r_{f}(t), \\
\xi_{3}=g_{3}\left(\frac{r-r_{f}(t)}{1-r_{f}(t)}\right) \quad \text { for } r_{f}(t)<r<1 .
\end{array}
$$

where $\xi_{j}=0$ corresponds to the left-hand boundary of the respective domain, and $\xi_{j}=1$ corresponds to the right, where $j \in\{1,2,3\}$. In $(54), g_{1}(\eta)=g_{3}(\eta)=\eta$, and $g_{2}(\eta ; \lambda)=-\log \left[1-\eta\left(1-e^{-\lambda}\right)\right] / \lambda$ is a monotonic increasing function, with $g_{2}(0)=0$ and $g_{2}(1)=1$. Here, $\lambda \in(0, \infty)$ is a constant we choose, with a larger value of $\lambda$ corresponding to a finer grid resolution near $r=r_{f}^{-}$with a uniform discretization of $\xi_{2}$. The limit $g_{2}(\eta ; \lambda) \rightarrow \eta$ is reached as $\lambda \rightarrow 0^{+}$. We show $g_{2}(\eta ; \lambda)$ in Figure 12 .

Under the transformations (54), the derivatives transform as follows

$$
\frac{\partial}{\partial r} \mapsto \frac{g_{j}^{\prime}\left[g_{j}^{-1}\left(\xi_{j}\right)\right]}{b_{j}-a_{j}} \frac{\partial}{\partial \xi_{j}}, \quad \frac{\partial}{\partial t} \mapsto \frac{\partial}{\partial t}-\frac{g_{j}^{\prime}\left[g_{j}^{-1}\left(\xi_{j}\right)\right]}{b_{j}-a_{j}}\left[\dot{a}_{j}\left(1-g_{j}^{-1}\left(\xi_{j}\right)\right)+\dot{b}_{j} g_{j}^{-1}\left(\xi_{j}\right)\right] \frac{\partial}{\partial \xi_{j}} .
$$

Therefore, the governing equations (9)-(10) are transformed as follows:

$$
\frac{\partial c_{i_{j}}^{j}}{\partial t}=\frac{g_{j}^{\prime}\left[g_{j}^{-1}\left(\xi_{j}\right)\right]}{b_{j}-a_{j}}\left[\dot{a}_{j}\left(1-g_{j}^{-1}\left(\xi_{j}\right)\right)+\dot{b}_{j} g_{j}^{-1}\left(\xi_{j}\right)\right] \frac{\partial c_{i_{j}}^{j}}{\partial \xi_{j}}+D_{i_{j}}^{j} \frac{g_{j}^{\prime}\left[g_{j}^{-1}\left(\xi_{j}\right)\right]}{b_{j}-a_{j}} \frac{\partial}{\partial \xi_{j}}\left(\frac{g_{j}^{\prime}\left[g_{j}^{-1}\left(\xi_{j}\right)\right]}{b_{j}-a_{j}} \frac{\partial c_{i_{j}}^{j}}{\partial \xi_{j}}\right)
$$

on the domain $0<\xi_{j}<1$. Here, $i_{1} \in\{1,2,3\}, i_{2} \in\{1,2,3\}$, and $i_{3} \in\{1\}$, and we note that $g_{1}(\eta)=g_{3}(\eta)=\eta$ greatly simplifies (56) in the cell and solid domains. We specify $a_{j}(t), b_{j}(t), c_{i_{j}}^{j}$, and $D_{i_{j}}^{j}$ in Table 4 .

The boundary conditions at the origin (11) become

$$
X_{c}=Y_{c}=\Theta_{l, c}=0 \quad \text { for } \xi_{1}=0,
$$




\begin{tabular}{|c|c|c|c|c|c|c|}
\hline$j$ & $g_{j}(\eta)$ & $a_{j}(t)$ & $b_{j}(t)$ & $i_{j}$ & $c_{i_{j}}^{j}$ & $D_{i_{j}}^{j}$ \\
\hline 1 & $\eta$ & 0 & $r_{c}(t)$ & $\begin{array}{l}1 \\
2 \\
3\end{array}$ & $\begin{array}{c}X_{c} \\
Y_{c} \\
\Theta_{l, c}\end{array}$ & $\begin{array}{c}D_{c}^{x} \\
D_{c}^{y} \\
k_{l}\end{array}$ \\
\hline 2 & $-\frac{1}{\lambda} \log \left[1-\eta\left(1-e^{-\lambda}\right)\right]$ & $r_{c}(t)$ & $r_{f}(t)$ & $\begin{array}{l}1 \\
2 \\
3\end{array}$ & $\begin{array}{c}X_{l} \\
Y_{l} \\
\Theta_{l, l}\end{array}$ & $\begin{array}{c}D_{l}^{x} \\
D_{l}^{y} \\
k_{l}\end{array}$ \\
\hline 3 & $\eta$ & $\begin{array}{l}r_{f}(t) \\
\text { 3LE } 4\end{array}$ & 1 & 1 & $\Theta_{s}$ & 1 \\
\hline
\end{tabular}

The boundary conditions at the cell membrane (12) become

$$
D_{c}^{x}\left(\frac{\partial X_{c}}{\partial \xi_{1}}-X_{c}\right)+X_{c} r_{c} \frac{\mathrm{d} r_{c}}{\mathrm{~d} t}=D_{l}^{x}\left(\frac{r_{c}\left(1-e^{-\lambda}\right)}{\lambda\left(r_{f}-r_{c}\right)} \frac{\partial X_{l}}{\partial \xi_{2}}-X_{l}\right)+X_{l} r_{c} \frac{\mathrm{d} r_{c}}{\mathrm{~d} t} \quad \text { for } \xi_{1}=1, \xi_{2}=0
$$$$
D_{c}^{x}\left(\frac{\partial X_{c}}{\partial \xi_{1}}-X_{c}\right)+X_{c} r_{c} \frac{\mathrm{d} r_{c}}{\mathrm{~d} t}=\omega r_{c}\left(X_{l}-X_{c}\right) \quad \text { for } \xi_{1}=1, \xi_{2}=0,
$$$$
D_{c}^{y}\left(\frac{\partial Y_{c}}{\partial \xi_{1}}-Y_{c}\right)+Y_{c} r_{c} \frac{\mathrm{d} r_{c}}{\mathrm{~d} t}=0 \text { for } \xi_{1}=1,
$$

$$
\begin{gathered}
D_{l}^{y}\left(\frac{r_{c}\left(1-e^{-\lambda}\right)}{\lambda\left(r_{f}-r_{c}\right)} \frac{\partial Y_{l}}{\partial \xi_{2}}-Y_{l}\right)+Y_{l} r_{c} \frac{\mathrm{d} r_{c}}{\mathrm{~d} t}=0 \quad \text { for } \xi_{2}=0, \\
\frac{\mathrm{d} r_{c}}{\mathrm{~d} t}=-\frac{\kappa}{r_{c}}\left[\sigma\left(X_{l}-X_{c}\right)+\left(Y_{l}-Y_{c}\right)\right] \text { for } \xi_{1}=1, \xi_{2}=0,
\end{gathered}
$$$$
\Theta_{l, c}=\Theta_{l, l} \text { for } \xi_{1}=1, \xi_{2}=0,
$$

$$
\frac{\partial \Theta_{l, c}}{\partial \xi_{1}}=\frac{r_{c}\left(1-e^{-\lambda}\right)}{\lambda\left(r_{f}-r_{c}\right)} \frac{\partial \Theta_{l, l}}{\partial \xi_{2}} \text { for } \xi_{1}=1, \xi_{2}=0 .
$$

The boundary conditions at the freezing front (13) become

$$
\begin{array}{r}
\Theta_{l, l}=\Theta_{s}=-\alpha X_{l} \quad \text { for } \xi_{2}=1, \xi_{3}=0, \\
D_{l}^{x}\left(\frac{r_{f}\left(e^{\lambda}-1\right)}{\lambda\left(r_{f}-r_{c}\right)} \frac{\partial X_{l}}{\partial \xi_{2}}-X_{l}\right)+X_{l} r_{f} \frac{\mathrm{d} r_{f}}{\mathrm{~d} t}=0 \quad \text { for } \xi_{2}=1, \\
D_{l}^{y}\left(\frac{r_{f}\left(e^{\lambda}-1\right)}{\lambda\left(r_{f}-r_{c}\right)} \frac{\partial Y_{l}}{\partial \xi_{2}}-Y_{l}\right)+Y_{l} r_{f} \frac{\mathrm{d} r_{f}}{\mathrm{~d} t}=0 \quad \text { for } \xi_{2}=1, \\
\operatorname{Sr}_{f}^{2} \frac{\mathrm{d} r_{f}}{\mathrm{~d} t}=\frac{r_{f}}{1-r_{f}} \frac{\partial \Theta_{s}}{\partial \xi_{3}}-\Theta_{s}-k\left(\frac{r_{f}\left(e^{\lambda}-1\right)}{\lambda\left(r_{f}-r_{c}\right)} \frac{\partial \Theta_{l, l}}{\partial \xi_{2}}-\Theta_{l, l}\right) \quad \text { for } \xi_{2}=1, \xi_{3}=0 .
\end{array}
$$

The boundary condition at the exterior ice boundary (15) becomes

$$
\Theta_{s}=T_{p}(t) \text { for } \xi_{3}=1
$$

Finally, the initial conditions (17) are now

$$
\begin{array}{r}
X_{c}\left(\xi_{1}, 0\right)=r_{c 0} \xi_{1}, \quad Y_{c}\left(\xi_{1}, 0\right)=Y_{0} r_{c 0} \xi_{1}, \quad \Theta_{l, c}\left(\xi_{1}, 0\right)=-\alpha r_{c 0} \xi_{1}, \\
X_{l}\left(\xi_{2}, 0\right)=r_{c 0}+\left(1-r_{c 0}\right) \frac{1-e^{-\lambda \xi_{2}}}{1-e^{-\lambda}}, \quad Y_{l}\left(\xi_{2}, 0\right)=Y_{0}\left(r_{c 0}+\left(1-r_{c 0}\right) \frac{1-e^{-\lambda \xi_{2}}}{1-e^{-\lambda}}\right), \\
\Theta_{l, l}\left(\xi_{2}, 0\right)=-\alpha\left(r_{c 0}+\left(1-r_{c 0}\right) \frac{1-e^{-\lambda \xi_{2}}}{1-e^{-\lambda}}\right), \quad r_{c}(0)=r_{c 0}, \quad r_{f}(0)=1 .
\end{array}
$$


We discretize our spatial operators using a standard second-order accurate central-difference scheme with uniform grid spacing. We discretize our boundary conditions using forward- or backward-difference schemes where appropriate, with second-order accuracy (verified in the supplementary material). We use 80 grid points in the cell and ice regions, and 300 grid points in the liquid region. For a given cooling rate, we choose $\lambda$ such that there are at least 10 points in the initial boundary layer identified in Appendix C. This ranged from $\lambda=10^{-5}$ for low cooling rates to $\lambda=8.7$ for the highest cooling rate of $\tilde{\beta}=10^{6} \mathrm{~K} \mathrm{~s}^{-1}$. For $\tilde{\beta}>0.5 \mathrm{~K} \mathrm{~s}^{-1}$, we stop the simulation at $t=10$, then restart it using $\lambda=0$ on a re-meshed uniform grid. This procedure enables us to generate a solution more quickly. We found that this approach was sufficient to ensure that our solutions were well resolved for all time. The excellent agreement between our numerical and asymptotic solutions (Figures 8-9) gives us confidence in our results.

\section{Appendix C. Early-time asymptotics.}

In this section we derive the early-time solutions for a linear temperature drop. This will allow us to start our simulations at a small but finite time, thus side-stepping the issue of creating the ice phase at $t=0$. While we have several natural small parameters in our system, the small parameters we use for this analysis are $t$ and $1-r_{f}(t)$, formally treating all other dimensionless parameters in the system as $O(1)$. We also note that the early-time limit of $\S 3.2$, the first important timescale in the problem, is equivalent to the small-time limit of the full system (i.e. the limits of $D_{l}^{x} \rightarrow 0^{+}$and $t \rightarrow 0^{+}$commute), confirming that we have correctly identified the earliest interesting timescale $t=O(1)$.

For ease of numerical implementation, it is simpler to determine the early-time solutions to the transformed system derived in Appendix B. However, we can also use the results of $\S 3.2$ to guide our analysis, noting that for early-time the ice temperature is constant in $r, x_{c}=1, y_{c}=Y_{0}$, and $r_{c}(t)=r_{c 0}$. In terms of the transformed variables

$$
\begin{aligned}
& X_{c}\left(\xi_{1}, t\right) \sim r_{c 0} \xi_{1}, \quad Y_{c}\left(\xi_{1}, t\right) \sim Y_{0} r_{c 0} \xi_{1}, \quad \Theta_{l, c}\left(\xi_{2}, t\right) \sim-\alpha r_{c 0} \xi_{1}, \\
& \Theta_{s}\left(\xi_{3}, t\right) \sim\left(r_{f}(t)+\left(1-r_{f}(t)\right) \xi_{3}\right) T_{p}(t), \quad r_{c}(t) \sim r_{c 0} .
\end{aligned}
$$

The more interesting problems are for $X_{l}, Y_{l}, \Theta_{l, l}$ and $r_{f}$; governed by (56), with boundary conditions (59a)-(59c) and initial conditions (61). At early time, $X_{l}, Y_{l}$, and $\Theta_{l, l}$ are close to their initial values, with a boundary layer near $\xi_{2}=1$. While we also have an analytic expression for $\Theta_{l, l}$, we note that the infinite sum in (22) requires many terms to evaluate accurately as $t \rightarrow 0^{+}$. Therefore, it is helpful to obtain a simplified version in this limit. Although Euler-Maclaurin summation can be used for this purpose, the steps required are fairly involved. A simpler method is to note that there is an early-time boundary layer near the interface, and to solve the relevant equations for $\Theta_{l, l}$ in the small- $t$ limit. Following this process, we note that the correct early-time scalings are $1-r_{f}=O\left(t^{3 / 2}\right), 1-\xi_{2}=O\left(t^{1 / 2}\right), X_{l}-X_{l}\left(\xi_{2}, 0\right)=O(t)$, $Y_{l}-Y_{l}\left(\xi_{2}, 0\right)=O(t)$, and $\Theta_{l, l}-\Theta_{l, l}\left(\xi_{2}, 0\right)=O(t)$. Seeking similarity solutions in the boundary layer, and noting that $g_{2}^{\prime}(1)=\left(e^{\lambda}-1\right) / \lambda$, the early-time (additive) composite solutions [46] are

$$
\begin{array}{r}
X_{l} \sim r_{c 0}+\left(1-r_{c 0}\right) \frac{1-e^{-\lambda \xi_{2}}}{1-e^{-\lambda}}+\frac{\beta t}{\alpha} F\left(\frac{\lambda\left(1-\xi_{2}\right)\left(1-r_{c 0}\right)}{\left(e^{\lambda}-1\right) \sqrt{4 D_{l}^{x} t}}\right), \\
Y_{l} \sim Y_{0}\left(r_{c 0}+\left(1-r_{c 0}\right) \frac{1-e^{-\lambda \xi_{2}}}{1-e^{-\lambda}}\right)+\frac{\beta Y_{0} t}{\alpha} \sqrt{\frac{D_{l}^{x}}{D_{l}^{y}}} F\left(\frac{\lambda\left(1-\xi_{2}\right)\left(1-r_{c 0}\right)}{\left(e^{\lambda}-1\right) \sqrt{4 D_{l}^{y} t}}\right), \\
\Theta_{l, l} \sim-\alpha\left(r_{c 0}+\left(1-r_{c 0}\right) \frac{1-e^{-\lambda \xi_{2}}}{1-e^{-\lambda}}\right)-\beta t F\left(\frac{\lambda\left(1-\xi_{2}\right)\left(1-r_{c 0}\right)}{\left(e^{\lambda}-1\right) \sqrt{4 k_{l} t}}\right), \\
r_{f}(t) \sim 1-\frac{4 \beta}{3 \alpha} \sqrt{\frac{D_{l}^{x}}{\pi}} t^{3 / 2}, \\
F(z)=\left(2 z^{2}+1\right) \operatorname{erfc} z-\frac{2 z}{\sqrt{\pi}} e^{-z^{2}},
\end{array}
$$

where $F(z)$ satisfies the following ODE

$$
F^{\prime \prime}+2 z F^{\prime}-4 F=0, \quad F(0)=1, \quad F(\infty)=0 .
$$


Although $Y_{l}$ satisfies a Neumann condition at the interface, its solution can be written in terms of $F$.

We note that the early-time results we derive in this Appendix generalize to any nonlinear prescribed temperature drop with initial velocity $T_{p}^{\prime}(0)=-\beta$, where $0<\beta<\infty$.

Appendix D. Boundary layer problems for $t=O(1)$.

To investigate the ice temperature and the concentration boundary layers over the timescale $t=O(1)$, we must account for the position of the freezing front as a small perturbation from its initial value. To this end, we introduce $R(t)=O(1)$, where

$$
r_{f}(t)=1-\sqrt{D_{l}^{x}} R(t) .
$$

Due to the slow movement of the freezing front over this timescale, the solute concentrations in the liquid phase take their initial values, $x_{l}=1$ and $y_{l}=Y_{0}$, in most of the liquid domain except within a boundary layer near the freezing front whose width is similar to that of the ice region. Therefore, we introduce the boundary layer coordinate

$$
\rho=(1-r) / \sqrt{D_{l}^{x}}-R(t),
$$

where $-R(t)<\rho<0$ corresponds to the ice region, $\rho=0$ corresponds to the freezing front, and $\rho>0$ corresponds to the liquid region boundary layer. Due to the ice phase being asymptotically small over this timescale, the leading-order problem for the liquid temperature will hold over the domain $0<r<1$.

Using the coordinate transform (66) for the ice region, the leading-order versions of the governing equations for the thermal problem (9) are

$$
\frac{\partial T_{l}}{\partial t}=\frac{k_{l}}{r^{2}} \frac{\partial}{\partial r}\left(r^{2} \frac{\partial T_{l}}{\partial r}\right) \quad \text { for } 0<r<1, \quad 0=\frac{\partial^{2} T_{s}}{\partial \rho^{2}} \quad \text { for }-R(t)<\rho<0 .
$$

The relevant leading-order boundary conditions are as follows. At the origin, we have the symmetry condition

$$
k_{l} \frac{\partial T_{l}}{\partial r}=0 \quad \text { for } r=0 .
$$

At the freezing front, we require the following conditions

$$
\left.T_{l}\right|_{r=1}=\left.T_{s}\right|_{\rho=0}, \quad \frac{\partial T_{s}}{\partial \rho}=0 \quad \text { for } \rho=0,
$$

where the first condition is continuity of temperature, and the second condition is a significantly reduced Stefan condition, essentially telling us that the ice phase is insulated to leading order at the freezing front. At the exterior ice boundary, we have

$$
T_{s}=T_{p}(t) \quad \text { for } \rho=-R(t)
$$

From the above system, we see that

$$
T_{s}=T_{p}(t),
$$

everywhere within the ice to leading order. Therefore, the coupling condition (69) yields the boundary condition (20).

The leading-order versions of the governing equations for the solute concentration problems (10) in the liquid region are

$$
\frac{\partial x_{l}}{\partial t}=\frac{\mathrm{d} R}{\mathrm{~d} t} \frac{\partial x_{l}}{\partial \rho}+\frac{\partial^{2} x_{l}}{\partial \rho^{2}}, \quad \frac{\partial y_{l}}{\partial t}=\frac{\mathrm{d} R}{\mathrm{~d} t} \frac{\partial y_{l}}{\partial \rho}+\frac{D_{l}^{y}}{D_{l}^{x}} \frac{\partial^{2} y_{l}}{\partial \rho^{2}} \quad \text { for } \rho>0,
$$

recalling the boundary layer coordinate given in (66). The relevant boundary conditions are

$$
x_{l}(1, t)=-T_{p}(t) / \alpha, \quad \frac{\partial x_{l}}{\partial \rho}+x_{l} \frac{\mathrm{d} R}{\mathrm{~d} t}=0, \quad \frac{D_{l}^{y}}{D_{l}^{x}} \frac{\partial y_{l}}{\partial \rho}+y_{l} \frac{\mathrm{d} R}{\mathrm{~d} t}=0 \quad \text { for } \rho=0 .
$$


To match into the outer liquid region, we have the conditions

$$
x_{l} \rightarrow 1, \quad y_{l} \rightarrow Y_{0} \quad \text { for } \rho \rightarrow \infty,
$$

Finally, the initial conditions of the system are as follows

$$
x_{l}(r, 0)=1, \quad y_{l}(r, 0)=Y_{0}, \quad R(0)=0 .
$$

From the above, we see that the motion of the freezing front is governed by the CPA concentration near the front, rather than from the release of heat due to freezing as one may have expected.

The reduced system for $x_{l}$ and $y_{l}$ near the freezing front is governed by (72)-(75). We note that the system for $y_{l}$ decouples from the problem for $x_{l}$ and $R$.

D.1. Very rapid cooling: $\beta \gg 1$. Using the fact that $T_{p} \rightarrow-1$ immediately for very rapid cooling, we can obtain analytic expressions for $x_{l}, y_{l}$, and $R$ over the conduction timescale. Seeking a similarity solution, we deduce

$$
y_{l} \sim Y_{0}\left\{\begin{array}{r}
\lambda x_{l} \sim 1+\left(\frac{1}{\alpha}-1\right) \frac{\operatorname{erfc}\left(\lambda+\frac{\rho}{2 \sqrt{t}}\right)}{\operatorname{erfc}(\lambda)}, \\
\lambda \sqrt{\frac{\pi D_{l}^{x}}{D_{l}^{y}}} \exp \left(\lambda^{2} \frac{D_{l}^{x}}{D_{l}^{y}}\right) \operatorname{erfc}\left(\sqrt{\frac{D_{l}^{x}}{D_{l}^{y}}}\left(\lambda+\frac{\rho}{2 \sqrt{t}}\right)\right) \\
\sqrt{\frac{\pi D_{l}^{x}}{D_{l}^{y}}} \exp \left(\lambda^{2} \frac{D_{l}^{x}}{D_{l}^{y}}\right) \operatorname{erfc}\left(\sqrt{\frac{D_{l}^{x}}{D_{l}^{y}}} \lambda\right)-1
\end{array}\right\},
$$

where $\lambda$ satisfies the transcendental equation

$$
\lambda \sqrt{\pi} \exp \left(\lambda^{2}\right) \operatorname{erfc} \lambda=1-\alpha,
$$

noting that $\alpha \in(0,1)$ from the definition of $\alpha$ (Table 3), since the initial dimensional temperature is $\tilde{T}_{0}:=\tilde{T}_{f 0}-\tilde{\alpha} \tilde{X}_{0}$.

\section{References.}

[1] D M Anderson, J D Benson, and A J Kearsley. Foundations of modeling in cryobiology-I: Concentration, Gibbs energy, and chemical potential relationships. Cryobiol, 69(3):349-360, 2014. doi: 10.1016/j.cryobiol.2014.09.004.

[2] D M Anderson, J D Benson, and A J Kearsley. Foundations of modeling in cryobiology-II: Heat and mass transport in bulk and at cell membrane and ice-liquid interfaces. Cryobiol, 2019. doi: 10.1016/j.cryobiol.2019. 09.014 .

[3] D M Anderson, J D Benson, and A J Kearsley. Foundations of modeling in cryobiology-III: Inward solidification of a ternary solution towards a permeable spherical cell in the dilute limit. Cryobiol, 2019. doi: 10.1016/j. cryobiol.2019.09.013.

[4] D M Anderson, J D Benson, and A J Kearsley. Numerical solution of inward solidification of a dilute ternary solution towards a semi-permeable spherical cell. Math Biosci, 316:108240, 2019. doi: 10.1016/j.mbs.2019.108240.

[5] J L Bailey, J-F Blodeau, and N Cormier. Semen cryopreservation in domestic animals: A damaging and capacitating phenomenon minireview. J Androl, 21(1):1-7, 2000.

[6] J D Benson, C C Chicone, and J K Critser. Analytical optimal controls for the state constrained addition and removal of cryoprotective agents. Bull Math Biol, 74(7):1516-1530, 2012. doi: 10.1007/s11538-012-9724-2.

[7] J D Benson, A J Kearsley, and A Z Higgins. Mathematical optimization of procedures for cryoprotectant equilibration using a toxicity cost function. Cryobiol, 64(3):144-151, 2012. doi: 10.1016/j.cryobiol.2012.01.001.

[8] J D Benson, C T Benson, and J K Critser. Mathematical model formulation and validation of water and solute transport in whole hamster pancreatic islets. Math Biosci, 254:64-75, 2014. doi: 10.1016/j.mbs.2014.06.003.

[9] J D Benson, A Z Higgins, K Desai, and A Eroglu. A toxicity cost function approach to optimal CPA equilibration in tissues. Cryobiol, 80:144-155, 2018. doi: 10.1016/j.cryobiol.2017.09.005. 
[10] A. Bensoussan, J.-L. Lions, and G. Papanicolaou. Asymptotic analysis for periodic structures. North-Holland Publishing Company, Amsterdam, 1978.

[11] J Dai, M P Sheetz, X Wan, and C E Morris. Membrane tension in swelling and shrinking molluscan neurons. J Neurosci, 18(17):6681-6692, 1998. doi: 10.1523/JNEUROSCI.18-17-06681.1998.

[12] M P Dalwadi, M Bruna, and I M Griffiths. A multiscale method to calculate filter blockage. J Fluid Mech, 809: 264-289, 2016. doi: 10.1017/jfm.2016.656.

[13] A F Davidson, J D Benson, and A Z Higgins. Mathematically optimized cryoprotectant equilibration procedures for cryopreservation of human oocytes. Theor Biol Med Model, 11(1):13, 2014. doi: 10.1186/1742-4682-11-13.

[14] A F Davidson, C Glasscock, D R McClanahan, J D Benson, and A Z Higgins. Toxicity minimized cryoprotectant addition and removal procedures for adherent endothelial cells. PloS one, 10(11):e0142828, 2015. doi: 10.1371/ journal.pone.0142828.

[15] R V Devireddy. Predicted permeability parameters of human ovarian tissue cells to various cryoprotectants and water. Mol Reprod Dev, 70(3):333-343, 2005. doi: 10.1002/mrd.20209.

[16] R V Devireddy, D J Smith, and J C Bischof. Effect of microscale mass transport and phase change on numerical prediction of freezing in biological tissues. J Heat Transf, 124(2):365-374, 2002. doi: 10.1115/1.1445134.

[17] G M Fahy. The relevance of cryoprotectant "toxicity" to cryobiology. Cryobiology, 23(1):1-13, 1986. doi: 10.1016/0011-2240(86)90013-1.

[18] B J Fuller. Cryoprotectants: the essential antifreezes to protect life in the frozen state. Cryoletters, 25(6): 375-388, 2004.

[19] S C Gupta. The classical Stefan problem: basic concepts, modelling and analysis, volume 45. Elsevier, 2003. doi: 10.1016/C2017-0-02306-6.

[20] M H Holmes. Introduction to perturbation methods, volume 20. Springer Science \& Business Media, 2012.

[21] W V Holt and A R Pickard. Role of reproductive technologies and genetic resource banks in animal conservation. Reviews of reproduction, 4(3):143-150, 1999. doi: 10.1530/ror.0.0040143.

[22] U. Hornung. Homogenization and porous media, volume 6. Springer Science \& Business Media, 2012.

[23] J S Ingwall and J A Balschi. Energetics of the na+ pump in the heart. J Cardiovasc Electrophysiol, 17:S127-S133, 2006. doi: 10.1111/j.1540-8167.2006.00397.x.

[24] A Jiao, X Han, J K Critser, and H Ma. Numerical investigations of transient heat transfer characteristics and vitrification tendencies in ultra-fast cell cooling processes. Cryobiol, 52(3):386-392, 2006. doi: 10.1016/j.cryobiol. 2006.01.009.

[25] J O M Karlsson and M Toner. Long-term storage of tissues by cryopreservation: critical issues. Biomat, 17(3): 243-256, 1996. doi: 10.1016/0142-9612(96)85562-1.

[26] O Kedem and A Katchalsky. Thermodynamic analysis of the permeability of biological membranes to nonelectrolytes. BBA, 27:229-246, 1958. doi: 10.1016/0006-3002(58)90330-5.

[27] J Kevorkian and J D Cole. Perturbation methods in applied mathematics, volume 34. Springer Science \& Business Media, 2013.

[28] S-J Kim, O D Bonner, and D-S Shin. Solvent-solute interactions in dimethylsulfoxide. J Chem Thermodyn, 3 (4):411-417, 1971. doi: 10.1016/S0021-9614(71)80024-1.

[29] F W Kleinhans. Membrane permeability modeling: Kedem-Katchalsky vs a two-parameter formalism. Cryobiol, 37(4):271-289, 1998. doi: 10.1006/cryo.1998.2135.

[30] H G Landau. Heat conduction in a melting solid. Q Appl Math, 8(1):81-94, 1950. doi: https://doi.org/10.1090/ qam/33441.

[31] S J Lee, L R Schover, A H Partridge, P Patrizio, W H Wallace, K Hagerty, L N Beck, L V Brennan, and K Oktay. American Society of Clinical Oncology recommendations on fertility preservation in cancer patients. J Clin Oncol, 24(18):2917-2931, 2006. doi: 10.1200/JCO.2006.06.5888.

[32] F Liu, S-S Zou, Y Zhu, C Sun, Y-F Liu, S-S Wang, W-B Shi, J-J Zhu, Y-H Huang, and Z Li. A novel microstraw for cryopreservation of small number of human spermatozoon. Asian J Androl, 19(3):326, 2017. doi: 10.4103/1008-682X.173452.

[33] P Mazur. Kinetics of water loss from cells at subzero temperatures and the likelihood of intracellular freezing. $J$ Gen Physiol, 47(2):347-369, 1963. doi: 10.1085/jgp.47.2.347.

[34] P Mazur. Cryobiology: the freezing of biological systems. Science, 168(3934):939-949, 1970. doi: 10.1126/ science.168.3934.939.

[35] P Mazur. Slow-freezing injury in mammalian cells. The freezing of mammalian embryos, 52:42-49, 1977. doi: 10.1016/0014-4827(72)90303-5.

[36] P Mazur. Equilibrium, quasi-equilibrium, and nonequilibrium freezing of mammalian embryos. Cell Biophys, 17 (1):53-92, 1990. doi: 10.1007/BF02989804.

[37] P Mazur. Principles of cryobiology. In Life in the frozen state, pages 29-92. CRC press, 2004. 
[38] P Mazur and J J Schmidt. Interactions of cooling velocity, temperature, and warming velocity on the survival of frozen and thawed yeast. Cryobiol, 5(1):1-17, 1968. doi: 10.1016/S0011-2240(68)80138-5.

[39] P Mazur, S P Leibo, and E H Y Chu. A two-factor hypothesis of freezing injury: evidence from Chinese hamster tissue-culture cells. Exp Cell Res, 71(2):345-355, 1972.

[40] P Mazur, K W Cole, J W Hall, P D Schreuders, and A P Mahowald. Cryobiological preservation of Drosophila embryos. Science, 258(5090):1932-1935, 1992. doi: 10.1126/science.1470915.

[41] D E Pegg. Principles of Cryopreservation. In Cryopreservation and Freeze-Drying Protocols, pages 33-45. CRC Press, 2009.

[42] C Polge, A U Smith, and A S Parkes. Revival of spermatozoa after vitrification and dehydration at low temperatures. Nature, 164(4172):666, 1949. doi: 10.1038/164666a0.

[43] W F Rall and G M Fahy. Ice-free cryopreservation of mouse embryos at $-196{ }^{\circ} \mathrm{C}$ by vitrification. Nature, 313 (6003):573, 1985. doi: 10.1038/313573a0.

[44] N Ray, T van Noorden, F Frank, and P Knabner. Multiscale modeling of colloid and fluid dynamics in porous media including an evolving microstructure. Transport in porous media, 95(3):669-696, 2012. doi: 10.1007/ s11242-012-0068-z.

[45] S Samuel Kim, H G Kang, N H Kim, H C Lee, and H H Lee. Assessment of the integrity of human oocytes retrieved from cryopreserved ovarian tissue after xenotransplantation. Human Reprod, 20(9):2502-2508, 2005. doi: 10.1093/humrep/dei099.

[46] M van Dyke. Perturbation methods in fluid mechanics. Academic Press New York, 1964.

[47] A S Verkman. Solute and macromolecule diffusion in cellular aqueous compartments. Trends Biochem Sci, 27 (1):27-33, 2002. doi: 10.1016/S0968-0004(01)02003-5.

[48] J Wolfe, M F Dowgert, and P L Steponkus. Mechanical study of the deformation and rupture of the plasma membranes of protoplasts during osmotic expansions. J Membrane Biol, 93(1):63-74, 1986. doi: 10.1007/ BF01871019.

[49] F Xu, S Moon, X Zhang, L Shao, Y S Song, and U Demirci. Multi-scale heat and mass transfer modelling of cell and tissue cryopreservation. Proc R Soc A, 368(1912):561-583, 2010. doi: 10.1098/rsta.2009.0248.

[50] K E Zachariassen. Physiology of cold tolerance in insects. Physiol Rev, 65(4):799-832, 1985. doi: 10.1152/ physrev.1985.65.4.799. 